ESJ Natural/Life/Medical Sciences

\title{
Sites D’eau Sacrés Et Conservation De La Biodiversité : Cas Des Paysages D’eau De La Région De Ziguinchor (Sénégal)
}

\author{
Mamadou Lamine Sané, \\ Doctorant à l'Ecole Doctorale Etudes sur l'Homme et la Société, \\ Département d'Histoire, Université Cheikh Anta DIOP de Dakar \\ Claudette Soumbane Diatta, \\ Département de Géographie, Université Cheikh Anta DIOP de Dakar \\ Barnabé Ephrem A. Diémé, \\ Laboratoire des Sciences et Techniques de l'Eau et de l'Environnement \\ (LaSTEE), Ecole Polytechnique de Thiès, Sénégal
}

Doi:10.19044/esj.2021.v17n43p18

Submitted: 29 September 2021

Accepted: 13 December 2021

Published: 31 December 2021
Copyright 2021 Author(s)

Under Creative Commons BY-NC-ND

4.0 OPEN ACCESS

\section{Cite As:}

Lamine Sané M.,Soumbane Diatta C., \& Ephrem A.D.B.,(2021). Sites d'eau sacrés et conservation de la biodiversité : cas des paysages d'eau de la région de Ziguinchor (Sénégal) European Scientific Journal, ESJ, 17(43), 18.

https://doi.org/10.19044/esj.2021.v17n43p18

\section{Résumé}

La région de Ziguinchor, située au sud du Sénégal, est entièrement comprise dans la Basse Casamance. Peuplée essentiellement par des Ajamaat, cette région se caractérise au plan géographique par une prédominance des paysages de l'eau qui lui donnent l'image d'un territoire « amphibie ». Cet environnement aquatique dominé par le fleuve Casamance d'où se détachent de nombreux bolong est aussi constitué de mangroves, de bas-fonds et de multitudes de mares qui leurs sont associées. Les populations ajamaat tirent ainsi une part essentielle de leurs ressources dans ce milieu. Toutefois, au-delà de son aspect nourricier, l'environnement aquatique occupe une place importante dans les croyances traditionnelles ajamaat. Considérés comme sanctuaires de nombreux êtres qui peuplent l'univers religieux ajamaat, les paysages de l'eau de la Basse Casamance sont des espaces sacrés dont l'accès est soumis à des interdits. Cet aspect traduit l'apport des croyances dans la préservation de la biodiversité.

Mots clés: Ajamaat, Basse Casamance, paysages de l'eau, sacré, croyances 


\title{
Sacred Water Sites And Biodiversity Conservation: The Case Of The Water Landscapes Of The Ziguinchor Region (Senegal)
}

\author{
Mamadou Lamine Sané, \\ Doctorant à l'Ecole Doctorale Etudes sur l'Homme et la Société, \\ Département d'Histoire, Université Cheikh Anta DIOP de Dakar \\ Claudette Soumbane Diatta, \\ Département de Géographie, Université Cheikh Anta DIOP de Dakar \\ Barnabé Ephrem A. Diémé, \\ Laboratoire des Sciences et Techniques de l'Eau et de l'Environnement \\ (LaSTEE), Ecole Polytechnique de Thiès, Sénégal
}

\begin{abstract}
The region of Ziguinchor, located in the south of Senegal, is entirely within Lower Casamance. Populated mainly by Ajamaat, this region is characterized geographically by a predominance of water landscapes that give it the image of an "amphibious" territory. This aquatic environment dominated by the Casamance River from which many bolongs stand out is also made up of mangroves, shallows and multitudes of ponds associated with them. The ajamaat populations thus draw an essential part of their resources from this environment. However, beyond its nourishing aspect, the aquatic environment occupies an important place in the traditional ajamaat beliefs. Considered as sanctuaries for many beings who populate the religious universe ajamaat, the water landscapes of Lower Casamance are sacred spaces whose access is subject to prohibitions. This aspect reflects the contribution of beliefs in the preservation of biodiversity.
\end{abstract}

Keywords: Ajamaat, Lower Casamance, Water Landscapes, Sacred, Beliefs

\section{Introduction}

Chaque société humaine demeure très largement tributaire du cadre de vie dans lequel elle puise la quasi-totalité de ses ressources. C'est pourquoi des relations particulières lient les sociétés au milieu naturel qui les entoure. A ce propos, Oyono et al. (2012, p.12) soulignent qu'en "Afrique Subsaharienne les rapports entre les sociétés humaines et l'environnement naturel sont le reflet d'une véritable homologie tissée par l'histoire, la géographie, les représentations du monde et les modes d'organisation sociale ». Cette situation peut se lire dans les rapports entre l'homme et son milieu physique en Basse Casamance. Région «amphibie », située au cœur des «rivières du sud », la Basse Casamance est en majorité peuplée par les 
Diolas encore appelés Ajamaat. Cet ethnonyme est usité par les populations pour désigner leur appartenance ethnique. En fait, le terme Diola, plus répandu, serait une attribution des autres groupes ethniques, notamment les Mandingues dans le contexte de violence qui avait marqué le contact des différents groupes en Sénégambie méridionale (Bassène, 2011 ; Diédhiou, 2011).

Les Bainounk ou Bañun, confinés aujourd'hui dans une trentaine de village autour de Ziguinchor et de Bignona, constitueraient cependant le peuplement le plus ancien de la Casamance. En effet, ils occupaient un vaste territoire au XVIe siècle, qui s'étendait de la rivière Cacheu en Guinée Bissau au marigot de Bintang (Gambie) (Linares, 1992). Mais leur prépondérance serait par la suite remise en question par les Ajamaat. Ces derniers auraient peuplé la Basse Casamance à partir des migrations venues du Sud (Pélissier, 1966 ; Baum, 1999). Ils ont d'abord peuplé le Huluf et le Esulalu sur la rive sud avant d'atteindre la rive nord (du fleuve Casamance) pour peupler le Jugut, le Karone et le Fogny (Thomas, 1960). Jusqu'au XIX ${ }^{\mathrm{e}}$ siècle, ces deux groupes constituaient la totalité de la population de la Basse Casamance (Pélissier P. 1966). Le dernier groupe dont la présence est assez remarquable au nord-ouest de la région est celui des Mandingues. Leur présence en Sénégambie serait antérieure au XIIIe siècle. Par la suite, ils vont fonder à partir du XVIIe siècle, le puissant royaume du Kaabu ou Gabou qui atteint son apogée au XVIII ${ }^{\mathrm{e}}$ siècle (Niane, 1989). Leur installation en Basse Casamance est assez tardive. Ce n'est qu'à partir du XIX ${ }^{\mathrm{e}}$ siècle, que de petits groupes longeant la frontière de Gambie pénétrèrent jusqu'aux environs de la côte (Pélissier, 1966). Ils constituent aujourd'hui une frange importante du Fogny Diabancounda et du Narang où ils cohabitent avec les Ajamaat.

Les Ajamaat sont tributaires des ressources naturelles tirées des milieux aquatiques. Ces derniers, composés de nombreux bolong rattachés au fleuve Casamance, de mares contigües aux rizières, de forêts de palétuviers, du littoral atlantique ont, depuis les époques de peuplement, constitué un intérêt vital pour ces populations. Mais, au-delà de cette importance économique, les paysages de l'eau en Basse Casamance se particularisent aussi par la sacralité qui les entoure. En effet, dans les représentations collectives, un point d'eau naturel, demeure rarement sans constituer un lieu de résidence des puissances surnaturelles qui peuplent l'univers religieux ajamaat. Les mânes des ancêtres, les êtres totémiques ainsi que divers génies qui peuplent le monde invisible ont pour habitacle privilégié, le milieu aquatique.

De ce fait, les mythes, les croyances associées à ces espaces, de même que les interdits qui régissent leur accès, ont constitué un moyen efficace de conservation de la biodiversité. Aujourd'hui, la Basse Casamance, à l'instar 
des autres régions d'Afrique occidentale, a connu à partir du XIX ${ }^{\mathrm{e}}$ siècle de profondes mutations culturelles dues à la pénétration coloniale et des religions dites révélées. Ce qui, inévitablement, a favorisé la désacralisation d'un grand nombre des paysages de l'eau et une altération des moyens traditionnels de conservation. La particularité ici, est le fait que les populations locales ont intégré certaines croyances populaires parvenues avec l'islamisation dans la sacralité des sites d'eau. Cette situation donna lieu à un véritable syncrétisme dans le rapport avec ces sites mais permet néanmoins d'inscrire leur préservation dans la longue durée.

Les travaux réalisés dans la zone d'étude sur les rapports entre croyances traditionnelles et paysages de l'eau (Fall et al., 2011 ; Oyono et al., 2012 ; Diatta et al., 2017 ; 2018 ; 2020) n'ont pas intégré l'aspect diachronique des représentations collectives sur les paysages d'eau en milieu ajamaat d'où l'intérêt de ce thème dans l'analyse des croyances et pratiques traditionnelles qui concourent à la sauvegarde de la biodiversité.

Cette étude est basée sur l'hypothèse selon laquelle, les représentations collectives des sociétés traditionnelles participent à la préservation de la biodiversité en dépit de l'avènement des religions étrangères (islam et christianisme) et des pressions anthropiques. Il s'agit de voir ici comment l'histoire peut aider à étudier les pratiques cultuelles et culturelles qui ont favorisé la préservation de l'environnement. A ce propos, la théorie de la génétique culturelle qui, à travers la globalité antérieure nous permet d'interroger les mémoires sur le contexte de l'initiative et de la création des méthodes de gestion de la biodiversité (Haenens 1989 cité par Sanou, 2003) est empruntée.

L'objectif assigné à cette étude est dans une première phase de revisiter les rapports entre les hommes et leur milieu proche, pour comprendre le processus qui a conduit à la nécessité de préserver la nature, particulièrement les paysages de l'eau. Enfin, mettre en exergue les éléments qui ont favorisé le maintien dans la longue durée de ces rapports privilégiés entre l’homme et la nature dans la société ajamaat.

\section{Lieu de l'étude}

Les paysages de l'eau ayant fait l'objet de cette étude concernent essentiellement le territoire de la Basse Casamance qui s'étend de l'océan Atlantique au Soungrougrou. Administrativement, la Basse Casamance correspond à la région de Ziguinchor. Elle est constituée par les départements d’Oussouye, de Ziguinchor et de Bignona. 


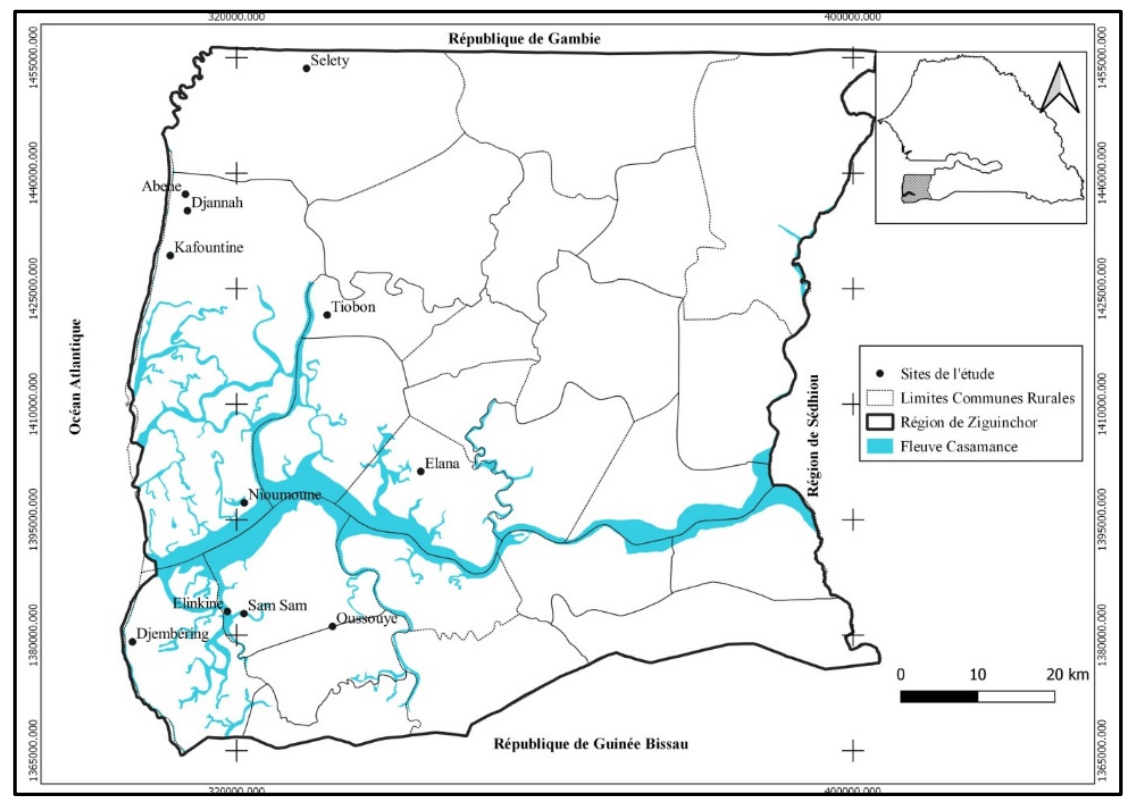

Carte 1 : Carte de situation

La population de la Basse Casamance, estimée à environ 641253 habitants, est constituée par une diversité d'ethnies dont les Ajamaat $(57,8 \%)$ qui cohabitent avec les Mandingue (11,1\%), les Pulaars (10,5\%), les Ouolofs (3,9\%), les Manjacques (3,5\%), les Balantes (2,9\%), les Mancagnes (2,4\%), les Sérères (2,7\%), etc. (ANSD/SRPSZ, 2015).

La région est très plate et s'intègre dans le bassin sédimentaire sénégalomauritanien. Elle est constituée de bas plateaux s'élevant à l'est et l'ouest du méridien de Bignona par des plateaux. Ces derniers surplombent les vallées peu encaissées qui s'ouvrent vers le fleuve ou l'Océan Atlantique. Vers l'embouchure, les bas-fonds cèdent la place à un paysage amphibie qui s'étend jusqu'à la dune côtière. Cette partie se caractérise par la présence des alluvions récentes au sein desquelles se rencontrent deux formations essentielles, d'une part des cordons de sable, d'autre part des plaines de vases couvertes de mangrove (Pélissier, 1966).

Le climat de la région est caractérisé par l'alternance d'une saison sèche (octobre-mai) et d'une saison humide (juin-septembre). La pluviométrie est supérieure à $1000 \mathrm{~mm} / \mathrm{an}$. L’hydrographie est marquée par la présence du fleuve Casamance auquel la région doit son histoire, son toponyme et ses principales ressources. Ce bras de mer est une rivière inverse qui coule d'ouest en est sur plus de $350 \mathrm{~km}$ (Diédhiou, 2001). Il est rattaché à un ensemble de marigots ou chenaux faisant des paysages d'eau un des éléments les plus caractéristiques de son milieu naturel et un patrimoine naturel et culturel remarquable pour les Ajamaat. 


\section{Démarche méthodologique}

La méthodologie suivie pour la réalisation du travail comporte deux phases, la documentation et la collecte des données qualitatives.

La phase de la documentation a consisté à consulter des archives historiques, des travaux universitaires et ouvrages sur la question des croyances traditionnelles développées par les sociétés autour des sites sacrés. Ces sources documentaires ont permis de faire l'état des lieux sur la question mais aussi d'orienter les guides d'entretien portant sur les savoirs et pratiques endogènes en rapport avec la préservation de l'environnement.

La collecte des données a principalement été faite au moyen d'un guide d'entretien et d'un questionnaire. La technique de "boule de neige » a été appliquée. Elle consiste à trouver un premier interlocuteur qui a conduit à un autre et ainsi de suite. Elle est appliquée à deux cibles principales :

- les personnes ressources : elles désignent les personnes disposant de suffisamment d'informations pour témoigner des pratiques traditionnelles et de leur évolution dans le temps. Il s'agit de chefs coutumiers, de dignitaires religieux et de patriarches dépositaires d'une certaine connaissance de la tradition. Après chaque entretien, la personne interviewée en désigne une autre avec qui le même travail est effectué.

- Les chefs de ménage : ils sont aussi associés à ce travail car constituant une source d'information non négligeable. En réalité, si les pratiques collectives tendent à disparaître, les familles conservent des résidus de celles-ci. Ainsi, un questionnaire leur a été soumis sous forme d'entretien individuel. Le but est de recueillir des informations afin de déceler la persistance ou non des pratiques traditionnelles locales. Les entretiens avec les chefs de ménages sont arrêtés dès constat du retour des mêmes réponses.

A la fin du processus, le comptage du nombre de personnes touché a été effectué et les résultats de celui-ci sont inscrits dans le tableau 1 par localité. Au total, 103 chefs de ménage et 44 personnes ressources sont interviewés dans le cadre de ce travail.

Le choix des villages est fondé sur l'existence de sites d'eau sacrés, leur répartition spatiale et des groupes linguistiques Ajamaat sans tenir compte de l'état de conservation des sites, du type de croyance (animistes, musulmanes), de leur activité ou abandon. Ainsi, sur la rive droite précisément au nord de la région de Basse Casamance, cinq villages ont été enquêtés à savoir Kafountine et Diannah situés dans le Fogny Diabancounda, Niomoune dans le Bliss-Kassa, Tiobon et Elana dans le Blouf. Sur la rive gauche (sud), six villages que sont Oussouye appartenant à la zone Kassa ou royaume du 
Houlouf, Sam Sam, Elinkine et Kagnoute (Esulalu), Djembéring (Diwat) et Séléky localisé dans le Bandial ou Mof Evvi ${ }^{1}$ ont été sélectionnés.

En plus des informations recueillies, les séjours sur le terrain d'enquête ont également permis d'effectuer des observations directes sur des faits et des pratiques en rapport avec la problématique de recherche.

Tableau 1 : Echantillonnage de la population enquêtée dans les villages de l'étude

\begin{tabular}{|c|c|c|}
\hline \multirow{2}{*}{ Localités/Villages } & \multicolumn{2}{|c|}{ Entretiens avec : } \\
\cline { 2 - 3 } & $\begin{array}{c}\text { Chefs de } \\
\text { ménage }\end{array}$ & $\begin{array}{c}\text { Personnes } \\
\text { ressources }\end{array}$ \\
\hline Kafountine & 10 & 3 \\
\hline Diannah & 15 & 7 \\
\hline Niomoune & 10 & 5 \\
\hline Tiobon & 15 & 5 \\
\hline Elana & 15 & 6 \\
\hline Oussouye & 10 & 3 \\
\hline Sam-sam & 5 & 3 \\
\hline Elinkine & 10 & 3 \\
\hline Djembéring & 8 & 6 \\
\hline Séléky & 5 & 3 \\
\hline Totaux & $\mathbf{1 0 3}$ & $\mathbf{4 4}$ \\
\hline
\end{tabular}

Source : Sané et al. 2021

\section{Résultats}

\subsection{Typologie des paysages d'eau sacrés de la Basse Casamance}

Les milieux aquatiques sont une composante essentielle de la géographie physique de la Basse Casamance et constituent une partie importante du patrimoine matériel et immatériel du peuple ajamaat. L’étude a permis d'inventorier plusieurs sites d'eau sacrés dans les différentes entités ajamaat de l'étude que sont le Fogny Diabancounda, le Bliss-Kassa, le Blouf, le Royaume du Houlouf, le Esulalu, la zone Diwat et le Bandial ou Mof Evvi. Ces sites se distinguent en fonction de leur spécificité (figure 1).

${ }^{1}$ Le Mof Evvi traduit littéralement par la «terre du roi » en langue ajamaat est un territoire bien structuré improprement appelé par les auteurs royaume du Bandial. Le Bandial en fait est un des six villages composant cette entité dont le nom traditionnel par lequel le désignent ses habitants est Esukuring « villages des rivières». 


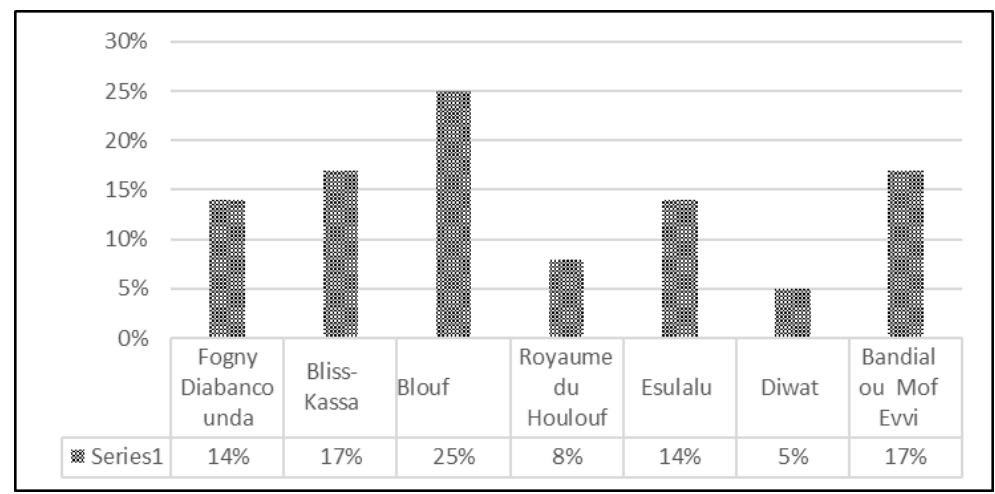

Figure 1 : Répartition des sites d'eau en fonction des entités traditionnelle ajamaat (Source : Sané et al. 2021)

La diversité des sites d'eau répertoriés rend difficile l'adoption d'un seul critère pour établir une typologie précise. C'est pourquoi une classification fondée sur les types de pratiques a été adoptée. Ainsi, quatre (04) types de sites d'eau sacrés se distinguent assez facilement : les sites d'eau animistes, les sites d'eau musulmans, les sites syncrétiques et sites abritant des djinns.

\subsubsection{Les sites d'eau liés à la religion traditionnelle ajamaat :}

Ce sont les plus répandues dans la zone et témoignent du dynamisme récent des pratiques traditionnelles. Ils se rapportent à ceux qui servent aux pratiques traditionnelles locales et sont constitués par des mares ou des bolong. On dénombre 23 sites d'eau sacrés strictement liés aux pratiques locales et animistes, répartis ainsi :

- 01 mare appelée foumodje en langue ajamaat considérée comme la demeure exclusive des mânes des ancêtres. Ce sont des points d'eau qui servent de pont entre la communauté et l'au-delà, celui de ses ancêtres. C'est pourquoi, ils requièrent une attention particulière de la part des populations ajamaat ;

- 13 mares et bolong abritant des totems (animaux) et dont l'exploitation des ressources est soumise à des régulations d'accès ou d'usage. ;

- 07 mares abritant indifféremment des totems, des génies, des mânes ancestrales et des lieux de rites cultuels ;

- 02 mares et bolong abritant exclusivement un fétiche

Tableau 2 : Sites d'eau liés aux pratiques animistes

\begin{tabular}{|c|c|c|c|c|c|}
\hline $\begin{array}{c}\text { Sites d'eau } \\
\text { sacrée }\end{array}$ & $\begin{array}{c}\text { Type de } \\
\text { paysage }\end{array}$ & $\begin{array}{c}\text { Raison de la } \\
\text { sacralité }\end{array}$ & $\begin{array}{c}\text { Pratiques } \\
\text { exécutées }\end{array}$ & Interdits & Villages \\
\hline Aroughy & Mare & $\begin{array}{c}\text { Usage } \\
\text { domestique } \\
\text { séculaire, } \\
\text { demeure } \\
\text { mânes des }\end{array}$ & $\begin{array}{c}\text { Rite } \\
\text { d'invocation } \\
\text { pour la pluie }\end{array}$ & $\begin{array}{c}\text { Accès } \\
\text { défendu aux } \\
\text { femmes en } \\
\text { menstrues ou }\end{array}$ & Tiobon \\
\hline
\end{tabular}




\begin{tabular}{|c|c|c|c|c|c|}
\hline $\begin{array}{l}\text { Sites d'eau } \\
\text { sacrée }\end{array}$ & $\begin{array}{l}\text { Type de } \\
\text { paysage }\end{array}$ & $\begin{array}{c}\text { Raison de la } \\
\text { sacralité }\end{array}$ & $\begin{array}{l}\text { Pratiques } \\
\text { exécutées }\end{array}$ & Interdits & Villages \\
\hline & & $\begin{array}{l}\text { ancêtres, lieu } \\
\text { de rite }\end{array}$ & & $\begin{array}{l}\text { accouchées } \\
\text { récentes }\end{array}$ & \\
\hline Colan & Mare & $\begin{array}{c}\text { Usage } \\
\text { domestique } \\
\text { séculaire, } \\
\text { demeure } \\
\text { mânes des } \\
\text { ancêtres, lieu } \\
\text { de rite }\end{array}$ & $\begin{array}{c}\text { Rites } \\
\text { initiatiques }\end{array}$ & $\begin{array}{c}\text { Accès } \\
\text { défendu aux } \\
\text { femmes en } \\
\text { menstrues ou } \\
\text { accouchées } \\
\text { récentes }\end{array}$ & Tiobon \\
\hline Djignilou & Mare & $\begin{array}{l}\text { Usage } \\
\text { domestique } \\
\text { séculaire, } \\
\text { présence de } \\
\text { totems }\end{array}$ & $\begin{array}{c}\text { Rites } \\
\text { initiatiques }\end{array}$ & $\begin{array}{c}\text { Interdit de } \\
\text { tuer les } \\
\text { animaux } \\
\text { vivant dans la } \\
\text { mare }\end{array}$ & $\begin{array}{l}\text { Mlomp } \\
\text { Blouf }\end{array}$ \\
\hline Kounankine & Bolong & $\begin{array}{l}\text { Abris de } \\
\text { totem }\end{array}$ & $X$ & $\begin{array}{c}\text { Interdit de } \\
\text { tuer les } \\
\text { animaux } \\
\text { vivant dans la } \\
\text { mare }\end{array}$ & $\begin{array}{l}\text { Mlomp } \\
\text { Blouf }\end{array}$ \\
\hline Kadieel & $\begin{array}{l}\text { Mare + } \\
\text { Forêt }\end{array}$ & $\begin{array}{c}\text { Usage } \\
\text { domestique } \\
\text { séculaire, } \\
\text { demeure des } \\
\text { mânes des } \\
\text { ancêtres, } \\
\text { présence de } \\
\text { génie titulaire } \\
\text { et de totems } \\
\end{array}$ & $\begin{array}{l}\text { Rite } \\
\text { d'invocation } \\
\text { aux mânes, au } \\
\text { génie et rite } \\
\text { d'initiation }\end{array}$ & $\begin{array}{c}\text { Accès } \\
\text { défendu aux } \\
\text { femmes en } \\
\text { menstrues ou } \\
\text { accouchées } \\
\text { récentes }\end{array}$ & Elana \\
\hline Ehouwa-assom & Mare & $\begin{array}{c}\text { Usage } \\
\text { domestique } \\
\text { séculaire, } \\
\text { demeure } \\
\text { mânes des } \\
\text { ancêtres, lieu } \\
\text { de rite } \\
\end{array}$ & $\begin{array}{c}\text { Rites } \\
\text { initiatiques }\end{array}$ & $\begin{array}{c}\text { Accès } \\
\text { défendu aux } \\
\text { femmes en } \\
\text { menstrues ou } \\
\text { accouchées } \\
\text { récentes }\end{array}$ & Niomoune \\
\hline $\begin{array}{c}\text { Kadiegue- } \\
\text { ajamaat }\end{array}$ & Mare & $\begin{array}{c}\text { Usage } \\
\text { domestique } \\
\text { séculaire, } \\
\text { demeure } \\
\text { mânes des } \\
\text { ancêtres, lieu } \\
\text { de rite }\end{array}$ & $\begin{array}{c}\text { Rite } \\
\text { d'invocation } \\
\text { pour la pluie }\end{array}$ & $\begin{array}{c}\text { Accès } \\
\text { défendu aux } \\
\text { femmes en } \\
\text { menstrues ou } \\
\text { accouchées } \\
\text { récentes }\end{array}$ & Niomoune \\
\hline
\end{tabular}




\begin{tabular}{|c|c|c|c|c|c|}
\hline $\begin{array}{l}\text { Sites d'eau } \\
\text { sacrée }\end{array}$ & $\begin{array}{l}\text { Type de } \\
\text { paysage }\end{array}$ & $\begin{array}{c}\text { Raison de la } \\
\text { sacralité }\end{array}$ & $\begin{array}{l}\text { Pratiques } \\
\text { exécutées }\end{array}$ & Interdits & Villages \\
\hline Outingue & $\begin{array}{c}\text { Bolong+ } \\
\text { mangrove }\end{array}$ & $\begin{array}{l}\text { Présence du } \\
\text { fétiche } \\
\text { Eniomounay }\end{array}$ & $\begin{array}{l}\text { Rite } \\
\text { d'invocation du } \\
\text { fétiche }\end{array}$ & $\begin{array}{c}\text { Protection } \\
\text { mystique des } \\
\text { ressources } \\
\text { assurée par le } \\
\text { fétiche } \\
\text { Eniomounay } \\
\end{array}$ & Niomoune \\
\hline $\begin{array}{l}\text { Hounak } \\
\text { Keumeuk }\end{array}$ & $\begin{array}{l}\text { Bolong+ } \\
\text { mangrove }\end{array}$ & $\begin{array}{l}\text { Abris de } \\
\text { totems }\end{array}$ & $\mathrm{X}$ & $\begin{array}{c}\text { Protection } \\
\text { des animaux } \\
\text { vivant dans la } \\
\text { mare }\end{array}$ & Niomoune \\
\hline Gnanguabitou & $\begin{array}{c}\text { Bolong+ } \\
\text { mangrove }\end{array}$ & $\begin{array}{l}\text { Abris de } \\
\text { totems }\end{array}$ & $\mathrm{X}$ & $\begin{array}{c}\text { Protection } \\
\text { des animaux } \\
\text { vivant dans la } \\
\text { mare }\end{array}$ & Niomoune \\
\hline Diahébéne & $\begin{array}{c}\text { Bolong+ } \\
\text { mangrove }\end{array}$ & $\begin{array}{l}\text { Abris de } \\
\text { totems }\end{array}$ & $\mathrm{X}$ & $\begin{array}{c}\text { Protection } \\
\text { des animaux } \\
\text { vivant dans la } \\
\text { mare } \\
\end{array}$ & Niomoune \\
\hline Guarem Bakène & Mare & $\begin{array}{l}\text { Usage } \\
\text { domestique } \\
\text { séculaire, } \\
\text { abris de } \\
\text { totems }\end{array}$ & $\begin{array}{c}\text { Rites de la } \\
\text { royauté du } \\
\text { Mofavi }\end{array}$ & $\begin{array}{c}\text { Accès } \\
\text { défendu aux } \\
\text { femmes en } \\
\text { menstrues ou } \\
\text { accouchées } \\
\text { récentes }\end{array}$ & Séléky \\
\hline $\begin{array}{l}\text { Djiousounguap } \\
\text { oh }\end{array}$ & Mare & $\begin{array}{c}\text { Usage } \\
\text { domestique } \\
\text { séculaire, } \\
\text { demeure des } \\
\text { mânes des } \\
\text { ancêtres } \\
\end{array}$ & $\begin{array}{c}\text { Rites } \\
\text { d'initiation, lieu } \\
\text { de réunion des } \\
\text { sages }\end{array}$ & $\begin{array}{c}\text { Accès } \\
\text { défendu aux } \\
\text { femmes en } \\
\text { menstrues ou } \\
\text { accouchées } \\
\text { récentes }\end{array}$ & Séléky \\
\hline Esoungar & Mare & $\begin{array}{l}\text { Usage } \\
\text { domestique } \\
\text { séculaire, } \\
\text { présence de } \\
\text { fétiche }\end{array}$ & $\begin{array}{c}\text { Rites } \\
\text { d'invocation au } \\
\text { fétiche } \\
\text { Batseumile de } \\
\text { Djoulounghène }\end{array}$ & $\begin{array}{c}\text { Accès } \\
\text { défendu aux } \\
\text { femmes en } \\
\text { menstrues ou } \\
\text { accouchées } \\
\text { récentes } \\
\end{array}$ & Séléky \\
\hline Fièques & $\begin{array}{c}\text { Bolong+ } \\
\text { mangrove }\end{array}$ & $\begin{array}{l}\text { Abris de } \\
\text { totems }\end{array}$ & $\mathrm{X}$ & $\begin{array}{c}\text { Protection } \\
\text { des animaux } \\
\text { vivant dans la } \\
\text { mare }\end{array}$ & Séléky \\
\hline Yeumeuh & $\begin{array}{c}\text { Bolong+ } \\
\text { mangrove }\end{array}$ & $\begin{array}{l}\text { Abris de } \\
\text { totems }\end{array}$ & $\mathrm{X}$ & $\begin{array}{c}\text { Protection } \\
\text { des animaux } \\
\text { vivant dans la } \\
\text { mare }\end{array}$ & Séléky \\
\hline
\end{tabular}




\begin{tabular}{|c|c|c|c|c|c|}
\hline $\begin{array}{l}\text { Sites d'eau } \\
\text { sacrée }\end{array}$ & $\begin{array}{l}\text { Type de } \\
\text { paysage }\end{array}$ & $\begin{array}{c}\text { Raison de la } \\
\text { sacralité }\end{array}$ & $\begin{array}{l}\text { Pratiques } \\
\text { exécutées }\end{array}$ & Interdits & Villages \\
\hline $\begin{array}{c}\text { Ewourouye ou } \\
\text { Gaora }\end{array}$ & $\begin{array}{l}\text { Bolong+ } \\
\text { mangrove }\end{array}$ & $\begin{array}{l}\text { Abris de } \\
\text { totems }\end{array}$ & $\mathrm{X}$ & $\begin{array}{c}\text { Protection } \\
\text { des animaux } \\
\text { vivant dans la } \\
\text { mare }\end{array}$ & Séléky \\
\hline Manel & Mare & $\begin{array}{l}\text { Usage } \\
\text { domestique } \\
\text { séculaire, } \\
\text { abris de } \\
\text { totems }\end{array}$ & $\begin{array}{l}\text { Eau servant de } \\
\text { libation au } \\
\text { fétiche de pluie } \\
\text { Kassarah }\end{array}$ & $\begin{array}{c}\text { Accès } \\
\text { défendu aux } \\
\text { femmes en } \\
\text { menstrues ou } \\
\text { accouchées } \\
\text { récentes } \\
\end{array}$ & Oussouye \\
\hline Diakokom & Mare & $\begin{array}{c}\text { Usage } \\
\text { domestique } \\
\text { séculaire, se } \\
\text { trouve dans } \\
\text { une rizière } \\
\text { consacrée au } \\
\text { fétiche } \\
\text { Dianande } \\
\end{array}$ & $\begin{array}{l}\text { Rizière réservée } \\
\text { au fétiche } \\
\text { Dianande }\end{array}$ & $\begin{array}{c}\text { Accès } \\
\text { défendu aux } \\
\text { femmes sauf } \\
\text { pour le } \\
\text { repiquage du } \\
\text { riz }\end{array}$ & Oussouye \\
\hline Etébrémouk & Mare & $\begin{array}{l}\text { Abris de } \\
\text { totems }\end{array}$ & $\mathrm{X}$ & $\begin{array}{c}\text { Protection } \\
\text { des animaux } \\
\text { vivant dans la } \\
\text { mare } \\
\end{array}$ & Oussouye \\
\hline Kassyssil & Mare & $\begin{array}{c}\text { Usage } \\
\text { domestique } \\
\text { séculaire, } \\
\text { demeure des } \\
\text { mânes des } \\
\text { ancêtres, } \\
\text { présence de } \\
\text { génie titulaire }\end{array}$ & $\begin{array}{c}\text { Rite } \\
\text { d'invocation } \\
\text { pour la pluie }\end{array}$ & $\begin{array}{l}\text { Accès } \\
\text { défendu aux } \\
\text { femmes en } \\
\text { menstrues ou } \\
\text { accouchées } \\
\text { récentes }\end{array}$ & Kagnoute \\
\hline Hono & $\begin{array}{l}\text { Bolong+ } \\
\text { mangrove }\end{array}$ & $\begin{array}{l}\text { Abris de } \\
\text { totems }\end{array}$ & $\mathrm{X}$ & $\begin{array}{c}\text { Protection } \\
\text { des animaux } \\
\text { vivant dans la } \\
\text { mare } \\
\end{array}$ & Kagnoute \\
\hline Katéhintémengh & $\begin{array}{c}\text { Bolong+ } \\
\text { mangrove }\end{array}$ & $\begin{array}{l}\text { Abris de } \\
\text { totems }\end{array}$ & $\mathrm{X}$ & $\begin{array}{c}\text { Protection } \\
\text { des animaux } \\
\text { vivant dans la } \\
\text { mare } \\
\end{array}$ & Kagnoute \\
\hline
\end{tabular}

(Source : Sané et al. 2021)

Il convient de retenir que les sites d'eau abritant des totems (animaux) sont les plus répandus (57\%). Ils sont suivis par les sites où l'on trouve à la fois les mânes des ancêtres, un génie ou des totems ou pratiques rituelles (30\%) et les sites avec un fétiche (13\%) (Figure 2). 


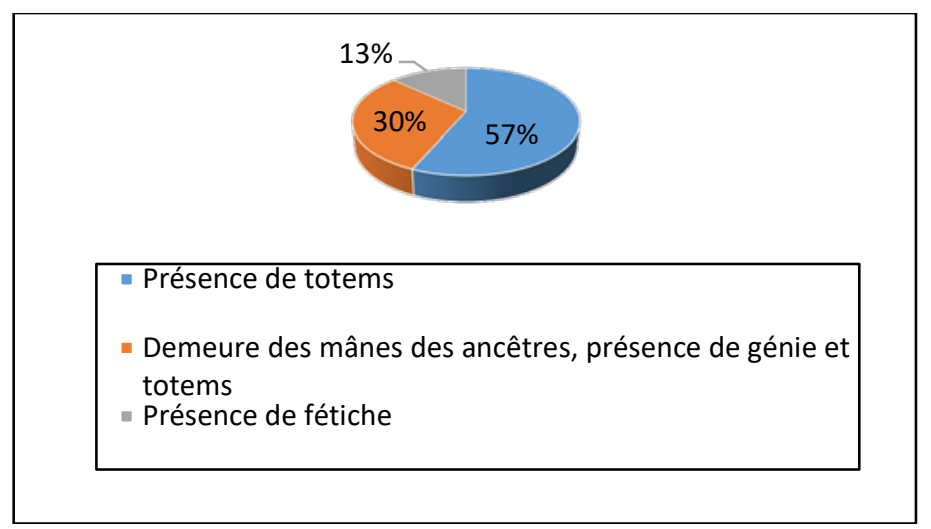

Figure 2 : Classification des sites liés aux pratiques animistes (Source : Sané et al. 2021)

\subsubsection{Les sites d'eau liés aux pratiques musulmanes :}

Il existe 4 sites d'eau sacrés dédiés aux pratiques musulmanes (tableau 3) et situés au nord de la zone d'étude. Il s'agit de la grotte de Boucotte-Diola, le puits d'El Hadj Omar à Elinkine, le puits Kegne kegne Diameng à Kafountine, la mare sacrée Dioulikaye à Sam Sam. L'existence de ces sites est liée au passage d'un érudit musulman. Ils font l'objet d'un pèlerinage de plus en plus intense. La bonne conservation de ces sites tranche le plus souvent avec la dégradation du proche environnement. Ces paysages d'eau sont composés de mares, de puits et de certains bolong. Les fidèles viennent y prendre des bains rituels pour conjurer le mauvais sort.

\subsubsection{Les sites syncrétiques}

Ils témoignent d'une une transition entre les croyances traditionnelles locales et musulmanes. Ils sont constitués par les mêmes objets géographiques (puis, mares, bolong). Les sites de cet ordre sont les mares sacrées de Barator à Elana, Nissaan à Diembéring (tableau 3). En effet, l'eau de ces sites qui jadis servait dans les pratiques cultuelles animistes a également été utilisée par le marabout pour s'abreuver et faire ses ablutions. Depuis, ces anciens sanctuaires animistes sont devenus un lieu de pèlerinage pour les musulmans de la zone.

Tableau 3 : Sites d'eau liés aux pratiques musulmanes et syncrétiques

\begin{tabular}{|c|c|c|c|c|c|c|}
\hline \multirow[b]{2}{*}{$\begin{array}{c}\text { Sites } \\
\text { musulmans }\end{array}$} & $\begin{array}{c}\text { Sites } \\
\text { d'eau } \\
\text { sacrée }\end{array}$ & $\begin{array}{l}\text { Type de } \\
\text { paysage }\end{array}$ & $\begin{array}{l}\text { Raison de } \\
\text { la sacralité }\end{array}$ & $\begin{array}{l}\text { Pratiques } \\
\text { exécutées }\end{array}$ & Interdits & Villages \\
\hline & $\begin{array}{c}\text { Kegne } \\
\text { kegne } \\
\text { Diameng }\end{array}$ & $\begin{array}{c}\text { Puits+ } \\
\text { forêt }\end{array}$ & $\begin{array}{c}\text { Lieu de } \\
\text { passage } \\
\text { d’El Hadj } \\
\text { Omar Tall }\end{array}$ & $\begin{array}{l}\text { Lieu de } \\
\text { prière et de } \\
\text { formulation } \\
\text { des vœux }\end{array}$ & $\begin{array}{c}\text { Défense } \\
\text { de } \\
\text { couper } \\
\text { les arbres }\end{array}$ & Kafountine \\
\hline
\end{tabular}




\begin{tabular}{|c|c|c|c|c|c|c|}
\hline & Dioulikaye & $\begin{array}{l}\text { Mare+ } \\
\text { forêt }\end{array}$ & $\begin{array}{c}\text { Lieu de } \\
\text { séjour d’El } \\
\text { Hadj Omar }\end{array}$ & $\begin{array}{l}\text { Utilisation } \\
\text { de l'eau } \\
\text { pour des } \\
\text { bains, des } \\
\text { ablutions et } \\
\text { lieu de } \\
\text { prière } \\
\end{array}$ & $\begin{array}{c}\text { Défense } \\
\text { de } \\
\text { couper } \\
\text { les arbres } \\
\text { du site }\end{array}$ & Sam Sam \\
\hline & $\begin{array}{c}\text { Teen-El } \\
\text { Hadj } \\
\text { Omar }\end{array}$ & Puits & $\begin{array}{l}\text { Existence } \\
\text { liée à El } \\
\text { Hadj Omar } \\
\text { Tall }\end{array}$ & $\begin{array}{l}\text { Utilisation } \\
\text { de l'eau } \\
\text { pour des } \\
\text { bains, des } \\
\text { ablutions et } \\
\text { lieu de } \\
\text { prière } \\
\end{array}$ & $X$ & Elinkine \\
\hline & $\begin{array}{l}\text { Dioulikaye } \\
\text { Izima }\end{array}$ & $\begin{array}{l}\text { Grotte } \\
\text { littorale }\end{array}$ & $\begin{array}{c}\text { Lieu de } \\
\text { séjours d’El } \\
\text { Hadj Omar }\end{array}$ & $\begin{array}{l}\text { Lieu de } \\
\text { prière et de } \\
\text { formulation } \\
\text { des vœux }\end{array}$ & $X$ & $\begin{array}{l}\text { Boucotte- } \\
\text { Diola }\end{array}$ \\
\hline \multirow{2}{*}{$\begin{array}{c}\text { Sites } \\
\text { syncrétiques }\end{array}$} & Barotor & Mare & $\begin{array}{c}\text { Usage } \\
\text { domestique } \\
\text { séculaire, } \\
\text { passage } \\
\text { d’El Hadj } \\
\text { Omar Tall }\end{array}$ & $\begin{array}{c}\text { Lieu de rite } \\
\text { en rapport } \\
\text { avec } \\
\text { l'initiation ; } \\
\text { lieu de } \\
\text { prière et de } \\
\text { formulation } \\
\text { de vœux } \\
\end{array}$ & $X$ & Elana \\
\hline & $\begin{array}{c}\text { Nissaan ou } \\
\text { Ekolong El } \\
\text { Hadj } \\
\text { Omar }\end{array}$ & Mare & $\begin{array}{c}\text { Usage } \\
\text { domestique } \\
\text { séculaire et } \\
\text { rituel, lieu } \\
\text { de séjours } \\
\text { d'El Hadj } \\
\text { Omar }\end{array}$ & $\begin{array}{l}\text { Utilisation } \\
\text { de l'eau } \\
\text { pour des } \\
\text { bains, des } \\
\text { ablutions, et } \\
\text { lieu de dépôt } \\
\text { d'offrande }\end{array}$ & $X$ & Djembéring \\
\hline
\end{tabular}

(Source : Sané et al. 2021)

\subsubsection{Les abris de génie ou djinn :}

Ils constituent un autre aspect du paysage des sites d'eau sacrés de la zone d'étude. Ils sont au nombre de sept et se retrouvent dans le Fogny Diabancounda et la zone Blouf (Tableau 4). La crainte de ces puissances surnaturelles entraine des attitudes d'évitement vis-à-vis de ces endroits et limite par la même le prélèvement de la ressource. 
Tableau 4 : Résidence de génie ou djinn

\begin{tabular}{|c|c|c|c|c|c|}
\hline $\begin{array}{l}\text { Sites d'eau } \\
\text { sacrée }\end{array}$ & $\begin{array}{l}\text { Type de } \\
\text { paysage }\end{array}$ & \begin{tabular}{|l|}
\multicolumn{2}{|l|}{ Raison } \\
der la \\
sacralité
\end{tabular} & $\begin{array}{l}\text { Pratiques } \\
\text { exécutées }\end{array}$ & Interdits & Villages \\
\hline Sounkoutoukala & $\begin{array}{l}\text { Bolong+ } \\
\text { mangrove }\end{array}$ & $\begin{array}{l}\text { Présence } \\
\text { d'un } \\
\text { génie } \\
\text { tutélaire }\end{array}$ & $\begin{array}{l}\text { Sacrifice } \\
\text { humaine } \\
\text { (abandonné) }\end{array}$ & $\begin{array}{l}\text { Protection } \\
\text { mystique des } \\
\text { ressources par le } \\
\text { génie }\end{array}$ & Diannah \\
\hline Dialomkoto & $\begin{array}{l}\text { Mare+ } \\
\text { forêt }\end{array}$ & $\begin{array}{l}\text { Présence } \\
\text { d'un } \\
\text { génie } \\
\text { tutélaire }\end{array}$ & $\begin{array}{l}\text { Rite d'invocation } \\
\text { au génie }\end{array}$ & $\begin{array}{l}\text { Accès défendu } \\
\text { sauf pour la } \\
\text { famille } \\
\text { dépositaire } \\
\text { (Diabang) }\end{array}$ & Diannah \\
\hline Doundala & $\begin{array}{l}\text { Mare+ } \\
\text { forêt }\end{array}$ & $\begin{array}{l}\text { Présence } \\
\text { d'un } \\
\text { génie } \\
\text { tutélaire }\end{array}$ & $\mathrm{X}$ & $\begin{array}{l}\text { Protection } \\
\text { mystique des } \\
\text { ressources par le } \\
\text { génie }\end{array}$ & Diannah \\
\hline Djinabantang & $\begin{array}{l}\text { Puits+ } \\
\text { forêt }\end{array}$ & $\begin{array}{l}\text { Présence } \\
\text { de djinn }\end{array}$ & \begin{tabular}{|lr} 
Pratiques & \\
initiatiques, lieu \\
de réunion & des \\
hommes & et \\
d'invocation & du \\
génie &
\end{tabular} & $\begin{array}{l}\text { Accès défendu } \\
\text { aux } \\
\text { femmes/défense } \\
\text { de couper les } \\
\text { arbres }\end{array}$ & Kafountine \\
\hline Doumbali & Bolong & $\begin{array}{l}\text { Présence } \\
\text { de djinn }\end{array}$ & Lieu de pêche & Aucun & Tiobon \\
\hline Erongol & Bolong & $\begin{array}{l}\text { Présence } \\
\text { de djinn }\end{array}$ & Lieu de pêche & Aucun & Tiobon \\
\hline Fussabe & Bolong & $\begin{array}{l}\text { Présence } \\
\text { de djinn }\end{array}$ & Lieu de pêche & Aucun & Tiobon \\
\hline
\end{tabular}

Suivant la classification établie ci-dessous, les sites liés à la religion traditionnelle ajamaat restent majoritaires avec 64\% des sites (tableau 5). Ce pourcentage élevé de sites animistes révèle l'encrage encore tenace des communautés dans les coutumes ancestrales surtout dans la partie sud de la région de Basse Casamance correspondant aux entités Houlouf, Bliss-Kassa, Esulalu, Diwat et Bandial. Les sites d'eau abritant les djinns représentent 19\% des sites sacrées et rend compte de la vivacité de de la croyance aux esprits. Par contre, les ceux liés aux pratiques musulmanes arrivent en $3^{\mathrm{e}}$ position et témoignent de la forte pénétration des valeurs islamiques. D’ailleurs, certains sites animistes sont transformés en lieu de pèlerinage ou de dévotion à un érudit musulman. Les sites syncrétiques constituent $6 \%$ des sites d'eau sacrés. La plupart sont en transition ou en voie d'abandon. 
Tableau 5 : Typologie des sites d'eau sacralisés de la zone d'étude

\begin{tabular}{|c|c|c|}
\hline Types de sites & Score & $\mathbf{\%}$ \\
\hline Les sites d'eau liés aux pratiques animistes & 23 & 64 \\
\hline Les sites d'eau liés aux pratiques musulmanes & 4 & 11 \\
\hline Les sites d'eau mixte syncrétiques (entre pratiques musulmanes et animistes) & 2 & 6 \\
\hline Résidence de génie ou djinn & 7 & 19 \\
\hline Total & 36 & 100 \\
\hline
\end{tabular}

(Source : Sané et al. 2021)

\subsection{Stratégies endogènes de protection et de préservation des paysages d'eau sacrés et de la biodiversité}

La protection et la gestion d'un certain nombre de paysages d'eau ont été, semble-t-il, une préoccupation majeure des Ajamaat. Ces lieux espaces d'eau sacralisés étaient et sont encore aujourd'hui pour la plupart régis par de nombreuses restrictions relatives à l'accès et l'exploitation des ressources. La limitation de l'accès de certains espaces à une catégorie de personnes notamment les non-initiés ou femmes encore en procréation, l'interdiction de prélèvement des ressources (coupe de bois, cueillette des huîtres, pêche, chasse d'animaux) sont entre autres des stratégies pour protéger ces sites d'eau sacrés. La violation des interdits qui entoure ces espaces peut donner lieu à des sanctions maléfiques de la part des puissances invisibles qui les protègent. Ainsi, ces interdits divers dont l'existence semble être liée aux croyances traditionnelles (culte des ancêtres, protection des totems, présence de fétiches où de génies), peuvent aussi être compris comme un moyen de protection de ces espaces.

\subsubsection{Paysages d'eau considérés comme résidence des ancêtres et des esprits des morts}

Les sites d'eau qui apparaissent comme des fukin ou demeure des mânes des ancêtres (Thomas L.V. 1959) sont pour l'essentiel, constitués de mares. Ces points d'eau étaient jadis utilisés par la population pour l'approvisionnement en eau. Mais ils demeurent sacrés dans de nombreux cas. Les ancêtres qui sont censés y demeurer sont des intermédiaires incontournables entre les divinités secondaires et la communauté. En général, chaque communauté ou lignage a un site considéré comme son fukin. Ces sites sont le plus souvent protégés par des interdits tels que l'accès aux femmes en période de menstruation.

Tableau 4 : Les mares abritant des rites dédiés aux mânes des ancêtres

\begin{tabular}{|c|c|}
\hline Mares & Localités \\
\hline Kadiégue ajamaat & Niomoune \\
\hline Ehouwa-Esoom & Niomoune \\
\hline Kadieel & Elana \\
\hline Colan & Tiobon \\
\hline
\end{tabular}




\begin{tabular}{|c|c|}
\hline Mares & Localités \\
\hline Aroughy & Tiobon \\
\hline Kassyssil & Kagnoute \\
\hline Djiousounguapoh & Séléky \\
\hline
\end{tabular}

(Source : Sané et al. 2021)

Par ailleurs, les cours d'eau (bolong et fleuve) et les forêts de palétuviers apparaissent dans l'imaginaire ajamaat comme étant protégés par les esprits de l'eau koummhāl (āmmhāl au singulier). Les koummhāl, esprits des morts, apparaissent comme maîtres des cours d'eau. Selon les croyances religieuses, ces esprits perçus comme des démons, protègent contre certaines pratiques abusives. En général, quand la pirogue d'un pêcheur chavire et que toute sa prise se déverse dans l'eau, on pense que ce fait découle de l'agissement des koummhāl. Ainsi l'existence de ces esprits dans les croyances ajamaat explique la crainte qu'inspirent les cours d'eau et le souci de ne pas susciter leur réaction négative en exploitant les ressources de façon incontrôlée.

\subsubsection{Paysages d'eau servant d'habitat aux totems}

Ils sont les plus nombreux et sont constitués de mares et de bolong (dont huit bolong et cinq mares). A noter que certains points considérés comme demeures des mânes abritent en même temps des totems. La croyance qui existe à leur égard se fonde sur la protection des doubles totémiques qui audelà, est une protection des membres de la communauté.

Concernant les mares, elles peuvent constituer des abris pour les crocodiles, des varans, des serpents, des silures, etc. Parmi ces mares, il y a Manel à Oussouye qui abrite des totems (varan) de la famille Diatta d'Oussouye. L'on raconte qu'autrefois, la mare de Manel ne tarissait pas même en saison sèche. De nos jours, lorsqu'elle tarit, du vin de palme y est versé en offrande avant de la recreuser. Le but de ce don est de demander aux totems de s'éloigner pour qu'ils ne soient pas touchés. La mare sacrée Guarem Bakène à Séléky abrite les totems de la famille Bassène. Elle sert aussi de point d'approvisionnement en eau. Son accès est interdit aux femmes en période de menstrues de même que celles qui viennent d'accoucher. Pour ces dernières, l'interdit n'est levé que lorsque l'enfant commence à pousser des dents. Il est en outre interdit d'y plonger un récipient en plastique ou d'y descendre avec des chaussures. Plusieurs autres mares considérées comme abris des totems ont été répertoriées dans la zone d'étude. Il s'agit entre autres du Kalamaho et du Djignilou à Mlomp, du Kadieel à Elana, du Djiousounguapoh et de l'Esoungar à Séléky, de l'Etébrémouk à Oussouye, etc.

Lorsque qu'il s'agit d'un bolong ou d'une rivière servant d'habitacle aux totems, les interdits visent en général la pêche et la cueillette des huîtres. C'est le cas par exemple à Niomoune avec les bolong de Hounak Keumeuk, Gnanguabitou et Diahébéne. 


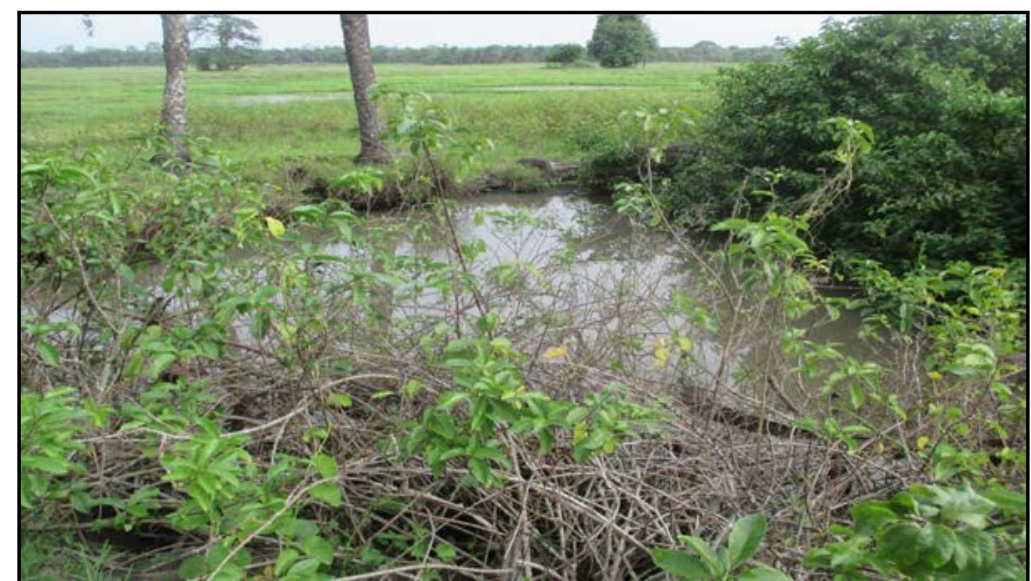

Photo 1 ; Mare sacrée de Manel abritant des totems reptiliens (Oussouye) (Source :

Sané et al., 2021)

\subsubsection{Paysages de l'eau protégés par un fétiche ou un génie tutélaire} A Niomoune, l'unique bolong Eniomounaye qui permet d'accéder à l'île à partir du fleuve Casamance est placé sous une protection mystique. En effet, dès l'entrée, le fétiche Eniomounaye (l'un des plus puissants et les plus craints dans la zone) qui sert d'hydronyme au bolong est implanté à gauche. Il semble que compte tenu de l'éloignement du village par rapport à ce site, les populations avaient le souci de protéger le village et les ressources du bolong (poissons, huitres, forêt de palétuviers...). Dans une société ajamaat où la croyance à ces puissances surhumaines reste vivace, la crainte qu'elles inspirent constitue un moyen de dissuasion efficace usité pour la protection de certains milieux.

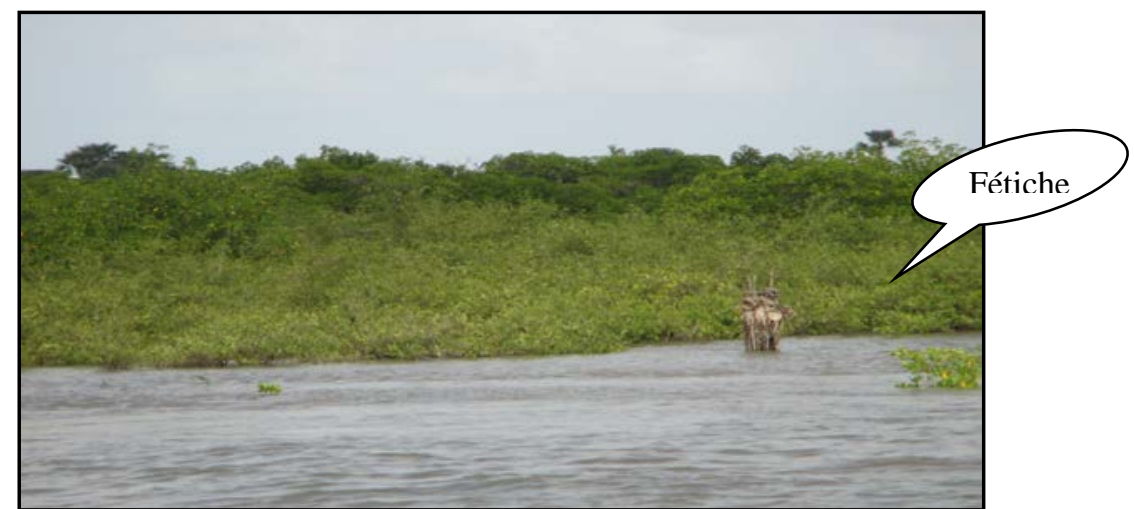

Photo 2 : Photo 2 : Boekin Eniomounay, bolong Niomoune (Source : Sané, 2018)

Quant à la mare Diakokom à Oussouye, elle est située dans une rizière sacrée appartenant au fétiche Dianande. Ce boekin protecteur d'Oussouye et de ses rizières est considéré comme l'un des plus puissants du royaume Huluf (Girard G.1969). Cette sacralité de la mare et de la rizière liée au boekin Dianande est 
la cause de l'interdiction de son accès aux femmes sauf pour la période de repiquage du riz dans ladite rizière. L'avantage écologique d'un tel site est le fait que tous les reptiles qui y élisent domicile sont épargnés par crainte d'offenser ce puissant boekin.

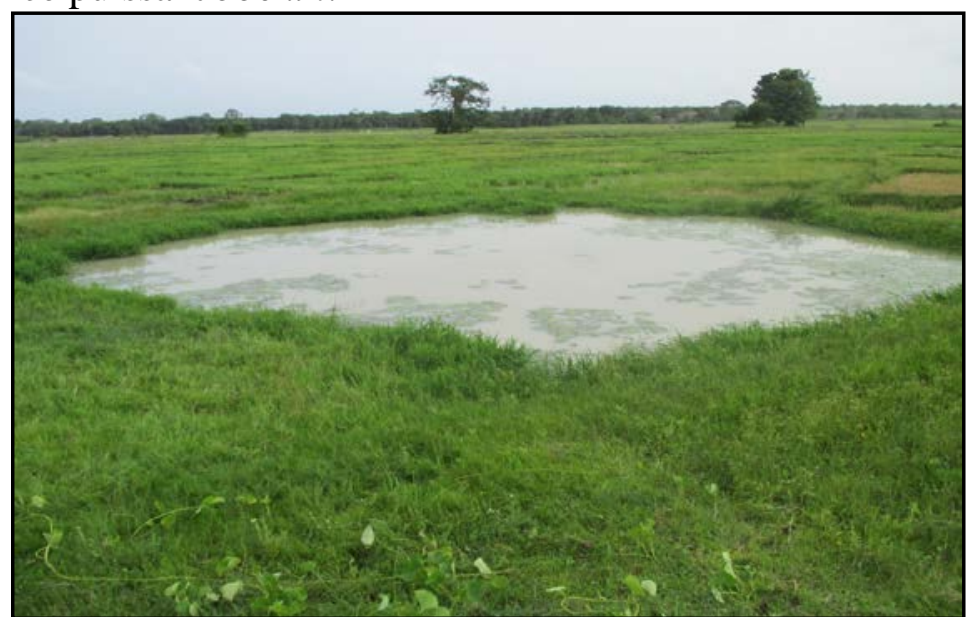

Photo 3 : Mare sacrée Diakokom située dans une rizière consacrée au boekin Dianande(Source : Sané, 2018)

Pour certains paysages de l'eau, des mythes ont été développés à propos du génie qu'ils abriteraient et qui suscite la crainte d’y pénétrer et exploiter des ressources. Parmi ces espaces, on peut citer le bolong de Sounkoutoukala à Diannah. Ce bolong qui a une disposition presque parallèle à la côte atlantique, est un passage obligé pour accéder à l'océan. Selon les croyances populaires, il constitue la demeure d'un génie qui se manifeste sous forme d'un miniyang$b a$ «gros serpent». Dans le passé, ce génie exigeait le sacrifice d’une jeune fille pour permettre le passage du bolong aux femmes qui partaient au ramassage des moules. C'est de ce sacrifice que vient l'hydronyme de Sounkoutoukala, qui signifie " fille pubère » dans la langue Mandingue. Le bolong, de même que la petite forêt qu'il traverse constituent un endroit très craint par la population à cause de la présence de ce génie. Ce qui confère au site un caractère intégré à l'approche traditionnelle de conservation de la biodiversité. La preuve est que le site abrite de nombreux reptiles comme les crocodiles, les serpents, les varans, etc. qui sont considérés comme protégés par le génie. On raconte dans le village «qu'un jeune du nom de Balla Moussa Bodian, s'adonnait à la chasse des crocodiles. Mais il tomba gravement malade et sa maladie fut interprétée comme relevant d'une sanction du génie du Sounkoutoukala. Aujourd'hui, il a cessé toute activité de chasse de ces crocodiles » (M T homme de 45ans ). Cette version des faits a d'ailleurs été confirmé par l'intéressé lui-même 


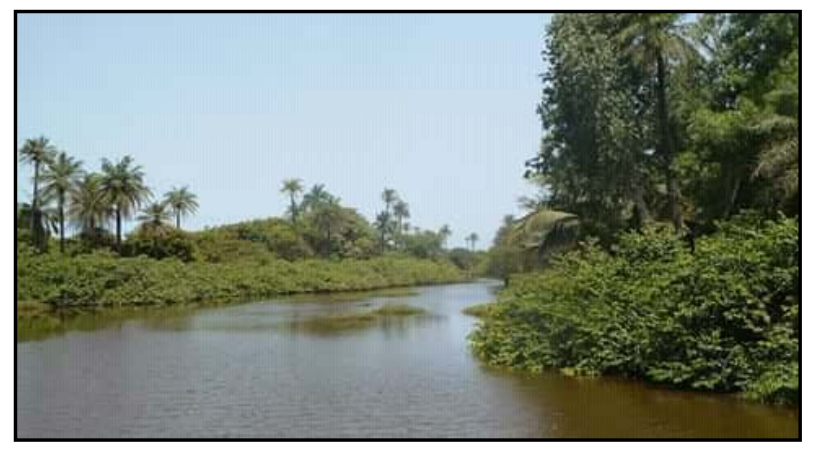

Photo 4 : Bolong de Sounkoutoukala à Diannah (Source : Sané et al. 2019)

Dans ce même village de Diannah, il y a la petite forêt de Dialomkoto située à proximité du bolong de Djindème ou Diannah bolong. Selon la tradition locale, c'est aux alentours de cette forêt que se seraient installés les ancêtres du clan des Diabang. Le site qui abrite une source d'eau qui se déversait dans le Diannah Bolong est la demeure d'un génie propriétaire des lieux. Les ancêtres des Diabang ont scellé un pacte avec ce génie pour résider dans le village. Seuls les membres de ladite famille, dépositaires des lieux, peuvent accéder librement à la forêt. Les autres personnes étrangères à cette famille devraient toujours être accompagnées par un de ses membres pour accéder à la forêt et faire des prélèvements (coupe ou chasse). Le génie qui apparait sous forme d'un gros serpent protège le village des malheurs éventuels. C'est pourquoi en cas de conflit avec un autre village, les sages hommes et femmes s'y rendent pour demander sa protection. Il s'agit d'un site très craint et protégé par peur de ne pas rompre le pacte ancestral scellé avec le génie et attirer des malheurs au village. Ainsi, malgré l'encrage de l'islam dans ce village et la pression foncière liée à l'implantation des vergers d'agrumes, cet espace est épargné.

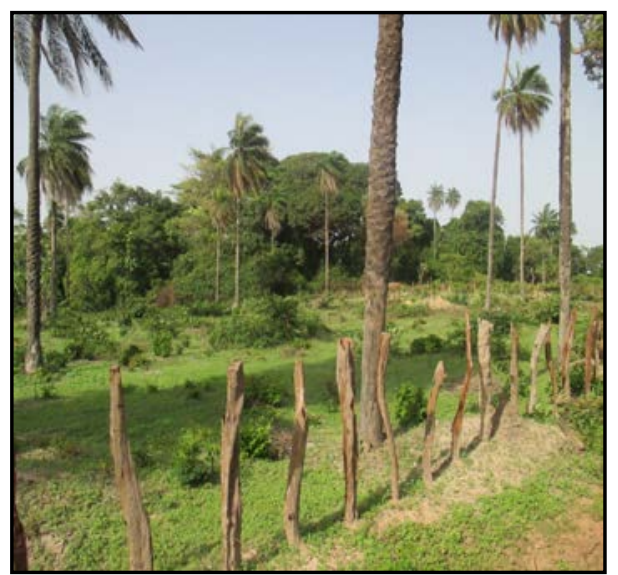

Photo 5 : Site de Dialomkoto (aménagement aux alentours, mais preservation de la forêt) (Source : Sané et al. 2019) 


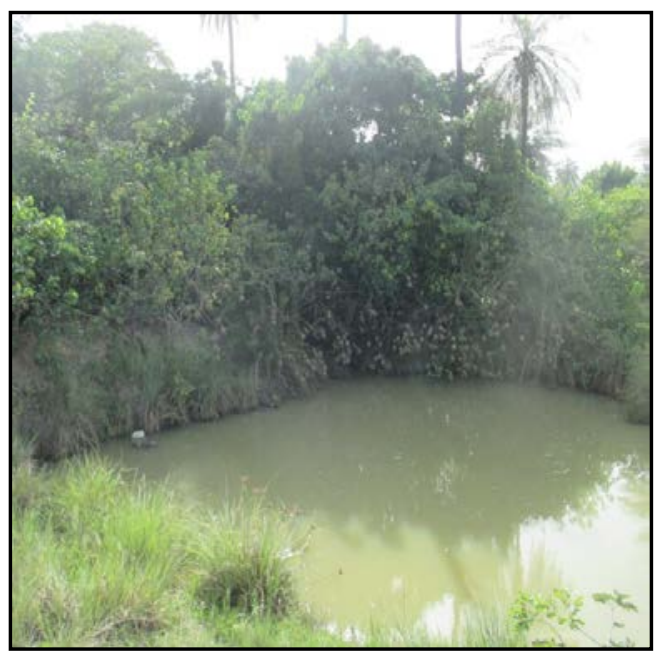

Photo 6 : Site de Diannah Bolong offrant une protection aux reptiles (Source : Sané et al. 2019)

Cette même croyance relative à un génie tutélaire ayant comme lieu de résidence un point d'eau est évoquée pour Doundala, un autre site à Diannah, Kadieel à Elana de même que Kassyssil à Kagnoute. Sur ces trois derniers sites, il existe de gros fromagers qui sont censés être la demeure du génie tutélaire des lieux. C'est pourquoi la sacralité du site concerne la mare et la végétation qui est aux alentours. Ainsi, pour Kadieel et Kassyssil, en plus des mânes des ancêtres sus mentionnés, les prières qui se font dans ces sites s'adressent en même temps au génie maître des lieux.

\subsubsection{Paysages d'eau sacralisés liés aux croyances islamiques}

Il s'agit dans un premier temps des sites considérés comme demeures des djinns. C'est le cas du puits de Djinabantang (ce terme signifie en mandingue «fromager des djinns ») à Kafountine, de Doumbali (« on n’y pénètre pas » en mandingue), de Erongol, et Fussabe endroits situés dans le bolong de Diouloulou vers Tiobon. L'accès et l'exploitation des ressources de ces sites qui sont pour l'essentiel des endroits spécifiques situés dans des bolong sont limités par la crainte des djinns qui y habitent.

Les autres points d'eau dont la sacralisation est liée aux croyances islamiques sont ceux en rapport avec le marabout El Hadj Omar Tall. En effet, dans le cadre de son djihad ce chef religieux musulman aurait effectué un périple qui l'a mené de la Gambie à la Guinée Bissau via la Basse Casamance et en longeant le littoral atlantique. Au cours de ce périple, le saint homme, qui vivait isolé, ne disposait pas d'eau pour ses ablutions. Il userait de son don mystique et surnaturel pour trouver de l'eau douce à côté du littoral ou des bolongs. Parmi ces points d'eau, il y a d'abord le puits de kegne kegne Diameng où il aurait séjourné en premier et qui se trouve à Kafountine au nord de la Basse Casamance à une trentaine de mètres de l'Océan atlantique. Le 
terme Kegne kegne Diameng signifie la "mosquée de sable” en référence à l'environnement immédiat constitué de sable marin. Ce lieu accueille un pèlerinage annuel de de fidèles venant de différentes régions du Sénégal, de la Gambie et d'autres pays voisins. Il est formellement interdit de fouler le sol de ce lieu sacré avec des chaussures ou d'y jeter des ordures.

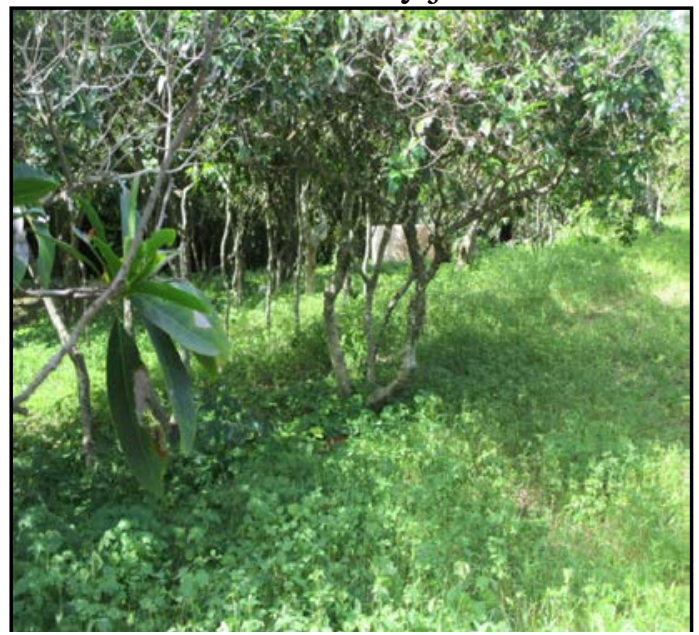

Photo 7 : Puits sacré de Kegne kegne diameng à Kafountine (Source : Sané et al., 2018)

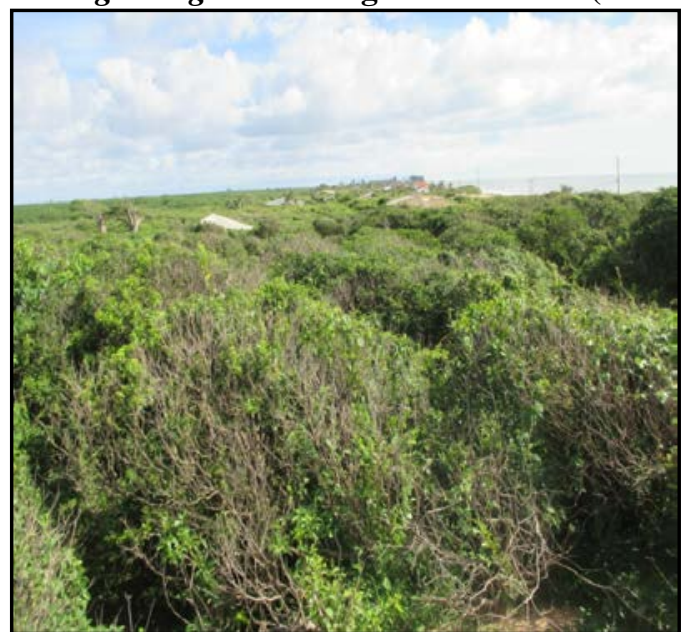

Photo 8 ; Vue aérien du site sacré de Kegne kegne diameng à Kafountine (Source :

Sané et al., 2018)

Outre le puits de Kegne kegne Diameng, il y a celui d'Elinkine ainsi que les mares d'eau douce de Barotor à Elana, de Sam Sam et de Nyssaan à Djembéring.

En fait, la mare sacrée de Barator à Elana aurait servi à Hadj Omar pour ses ablutions durant son séjour dans cette localité. Ignorée par les populations, c'est après le départ du marabout que son identité serait connue. Depuis lors, le site est devenu un lieu de culte où les gens viennent prier pour s'attirer les 
grâces divines. Des offrandes constituées de pièces d'argent, du lait caillé ou de galettes de riz y sont très souvent déposées par les visiteurs.

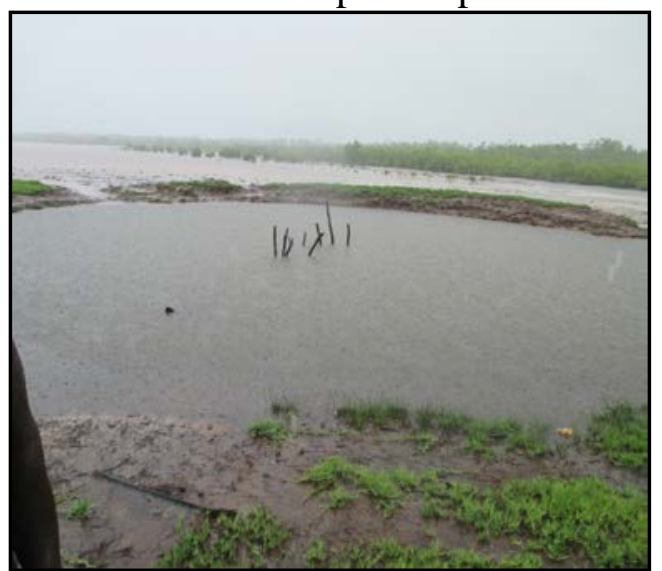

Photo 9 : Barotor, mare sacrée d’El Hadj Omar à Elana (Source: Sané et al., 2019)

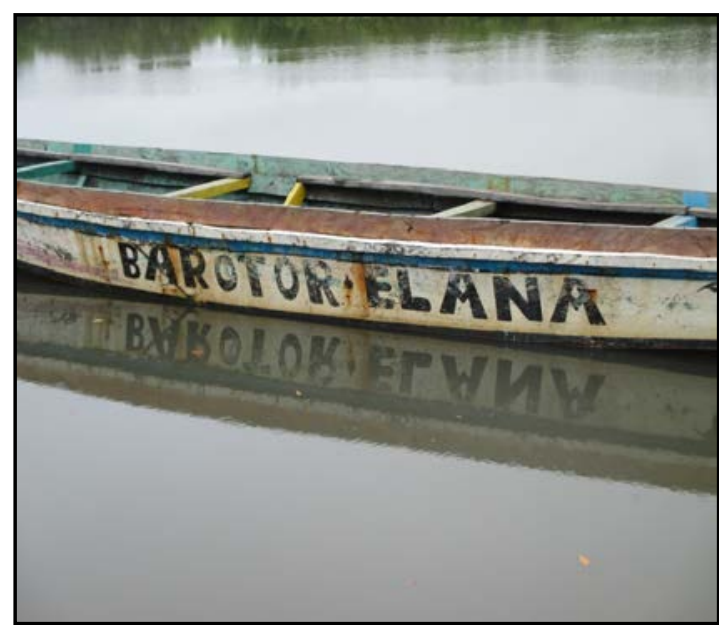

Photo 10 : Pirogue de liaison Elana Ziguinchor portant un hydronyme de mare sacrée pour des fins de protection (Source : Sané et al., 2019)

Sur la rive gauche de la Casamance, on retrouve la source de Sam Sam située à une dizaine de mètres de Oubaloum bolong. Cette source se trouve à l'intérieur d'une forêt qui est protégée. Le puits Elinkine, est quant à lui situé au niveau de l'embarcadère. Tout comme à Sam Sam, le marabout se serait arrêté sur ce lieu pour faire ses ablutions. Ce qui explique la présence de ce puits d'eau douce près d'un bolong dont les eaux sont salées.

Le saint-homme aurait par la suite continué son périple pour atterrir à Djembéring où il campa près d'une mare qui existait déjà et qui servait de lieu d'approvisionnement en eau pour la population. Les femmes qui partaient puiser l'eau de la mare le virent et lui apportèrent à manger durant tout son séjour. La mare est devenue depuis lors un lieu d'attraction où les gens 
viennent prier, déposer des offrandes et même prendre un bain purificateur pour bénéficier des bénédictions du saint homme.

De Djembéring, El Hadj Omar Tall poursuivit sa route vers le Sud et séjourna à Boucotte-Djembéring dans une grotte située sur le littoral et obtenait de l'eau pour ses ablutions dans une roche aujourd'hui envahit par la mer. Ce site, très protégé, devenu aussi un lieu de pèlerinage pour les fidèles serait le dernier endroit où séjourna le dignitaire musulman avant de partir pour la Guinée Bissau (Photo 11 et 12).

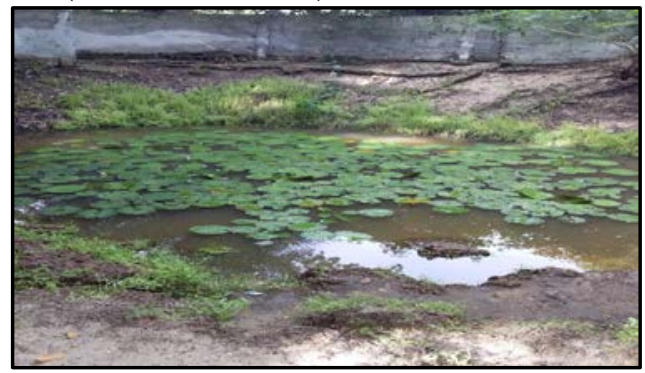

Photo 11 : Mare sacrée d'El Hadj Omar à Djembéring (Source : Sané et al., 2019)

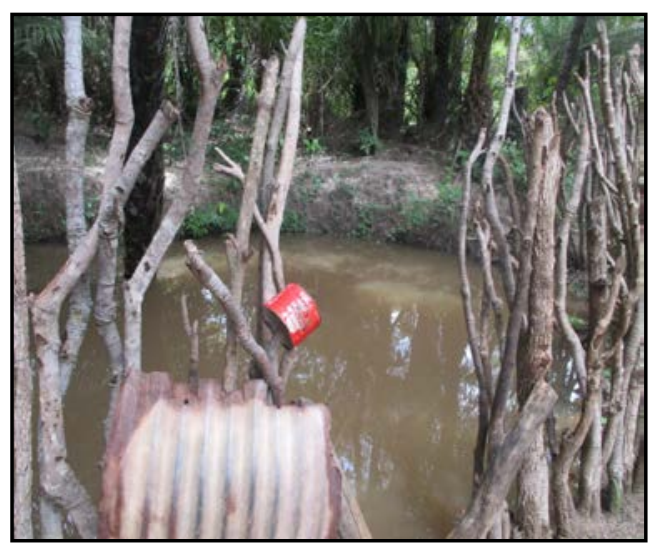

Photo 12 : Mare sacrée d’El Hadj Omar à Sam Sam (Source : Sané et al. 2019)

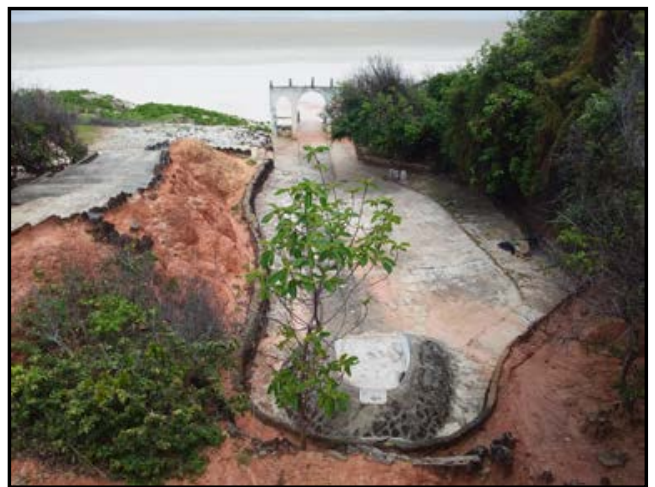

Photo 13 : Grotte sacré d'El Hadj OmarTall à Boucotte-Djembéring située en face de la mer (Source : Sané et al. 2019) 
Avec le passage du marabout, l'eau de ces sites est devenue bénite et les vœux formulés à ces endroits se réalisent. Il faut toutefois noter que dans certains sites comme Barotor à Elana et Nyssan à Djembéring, existaient déjà avant le passage d'El Hadj Omar et abritaient même des rites. Barotor est un passage obligé pour les futurs initiés avant d'entrer dans le bois sacré. Nyssan est une mare dont l'eau était utilisée pour la purification des veuves dans le cadre des rites de deuil chez les ajamaat Diwat. Ce qui dénote une fois de plus le déplacement du sacré opéré par les ajamaat pour continuer à préserver les paysages d'eau sacrés

\section{Discussion}

La religion traditionnelle ajamaat, à l'instar de la plupart des religions négro-africaines, place le milieu naturel au centre de la préoccupation des hommes. L'importance de ce milieu dans la vision du monde des ajamaat explique le caractère sacré de nombreux sites notamment dans l'environnement aquatique. Cet aspect confère une fonction écologique à cette religion traditionnelle en ce sens qu'elle réglemente l'accès et la gestion de bon nombre des paysages de l'eau en Basse Casamance. En effet, la protection à la fois humaine et mystique dont bénéficient ces sites, les transforme en sanctuaires pour de nombreuses espèces animales et végétales rares ou absentes dans les milieux non protégés (Butare 2003 ; Diatta et al. 2017). Ce rapport, empreint de respect et de crainte entre l'homme et l'environnement aquatique, a pu s'inscrire dans la longue durée à travers un syncrétisme entre les croyances locales et islamiques. Le syncrétisme religieux en milieu ajamaat est d'ailleurs à l'image de la société sénégalaise et africaine de façon générale (Quéchon, 1971 ; Diallo, 2009² ; Tabard, 2010). Tabard (2010, p. 193) écrit à ce sujet que "l'islam a trouvé, à travers les siècles, une identité spéciale qui fait qu'un Sénégalais ou un Malien musulman vit pleinement sa religion, tout en étant imprégné de sa culture négro-africaine». C’est également le cas pour le chrétien qui ne perd pas totalement sa culture "d'Homme et d'Africain" en recevant le baptême. Cela veut dire tout simplement que le baptême, "rite d'entrée dans l'Église conduit à parler d'une double identité ou d'une double appartenance : un africain baptisé appartient à la religion catholique tout en restant bien souvent profondément marqué, dans son identité, par sa culture africaine, voire par sa religion traditionnelle " (Tabard 2010, p. 192). En outre, pour parler comme Diallo (2009), ce qui est vrai pour l'islam l'est aussi pour le christianisme, car ils sont tous deux incultures dans les valeurs culturelles sénégalaises.

2 Diallo Amadou, «Syncrétisme et subversion religieuse dans Guelwaar de Sembène Ousmane », Ethiopiques n82. Littérature, philosophie, art et pluralisme, 1er semestre 2009. Article publié sur http://ethiopiques.refer.sn 
Le type de point d'eau qui, dès le départ, entra dans les préoccupations des premières communautés ajamaat fut sans doute la mare (foumodje), qui a servi de lieu d'approvisionnement en eau dans le passé. En effet, dans ce milieu " amphibie ", caractérisé par la présence de mares, de sources d'eau potable ou des affleurements de nappe phréatique, il semble que les populations ne foraient pas de puits mais se servaient de ces mares pour leurs besoins quotidiens en eau. Les traditions recueillies dans la zone d'étude rapportent que les premiers occupants et fondateurs desdits villages s'étaient installés autour des mares pour s'assurer de la disponibilité de l'eau en toute saison. Ceci prouve la préoccupation première qui prédomine dans toute idée d'installation d'une communauté humaine sur un espace, celle de disposer de l'eau qui est une source de vie. Toutefois, en plus de cette fonction de maintenir la vie, l'eau représente aussi un moyen de purification. Cette représentation symbolique de la ressource eau à proprement parlé se rencontre en Egypte pharaonique et dans les traditions négro-africaines les plus anciennes (Bonhême, 1995 ; Talkeu-Tounouga, 2000). Ainsi, il convient vraisemblablement de remarquer que dans l'Egypte ancienne "l'édification d'un temple qui a pour modèle l'émergence du monde hors des eaux, prend en compte la proximité de l'eau voire le contact de l'édifice avec l'élément liquide, qu'il s'agisse du fleuve par son canal ou encore des eaux phréatiques ressenties comme celles du Noun, l'océan primordiale " (Bonhême, 1995, pp.130-131). Ces lacs sacralisés servaient de lieux de purification des prêtres avant leur entrée dans le temple selon Bonhême (1995).

Par ailleurs, au-delà de sa fonction fondamentale pour la vie, l'eau symbolise la fécondité chez les Dogons du Mali ou est utilisée pour la bénédiction par le Bamileke du Cameroun (Talkeu-Tounouga, 2000). Chez ce même peuple Bamileke, Assipolo et Ndonko (2012) signalent l'importance de l'eau dans la pratique de l'ordalie, rite dans lequel il faut en réalité comprendre le cours d'eau avec toutes ses composantes. L'ordalie est une "Épreuve judiciaire employée au Moyen Âge pour établir l'innocence ou la culpabilité de l'accusé » (Assipolo et Ndonko, 2012, p. 4).

En milieu ajamaat la sacralisation des points d'eau serrait donc, dans un premier temps, liée à cette fonction importante de survie au cours des périodes antérieures. Dans la religion ajamaat, le contact avec la souillure du sang des menstrues est gnei-gnei ${ }^{3}$ (« interdit ») notamment pour les hommes. C'est pourquoi, pendant la période de leur menstruation, les femmes doivent s'abstenir de toute forme d'action qui les mettrait en contact avec la communauté, et observer des interdits comme celui lié à l'accès aux mares foumodje (Diatta, 2018). Ceci pour éviter de souiller ce point d'eau dont

\footnotetext{
${ }^{3}$ Gnei-gnei ou ñiñi selon les dialectes diola marque le sacré ou l'interdit. Il fait référence à quelque chose qui est taboue ou prohibée
} 
l'utilité est publique. Chez certaines sociétés négro-africaines où le puits a joué un rôle identique à la mare chez les Ajamaat, on lui confère la même sacralité. Chez les Tanalana du Madagascar, les vovon-draza, puits creusés par les ancêtres et leurs alentours ne doivent en aucun cas être souillées, car ils abritent des rituels et les locaux y puisent de l'eau (Tahirindraza, 2015).

Chez les Ajamaat, le respect voué à certains points d'eau est aussi lié au fait qu'ils soient considérés comme demeures privilégiés des mânes des ancêtres et des puissances surnaturelles. En effet, à l'image de la plupart des religions traditionnelles d'Afrique noire, le monde de l'au-delà ajaamat se localise au fond des eaux (Holas B., 1954 cité par Journet 1976 ; Zahan, 1956. Selon Journet (1976 ; p.99) « cette eau, d'obédience chtonienne est celle qui relie le monde des ancêtres aux vivants, c'est un monde de pénombre où se tiennent aussi les "ahuwa " [c'est-à-dire l'âme d'un ancêtre sur le point de se réincarner] ». C'est en rapport avec cette croyance, qu'en Basse Casamance beaucoup de points d'eau sont considérés comme des fukin (Diédhiou, 2001). Or les ancêtres qui sont censé y demeurer sont sollicités par leurs descendants pour porter leurs offrandes et intercéder en leur faveur auprès des puissances religieuses (Thomas, 1967). C'est pourquoi ils jouent une fonction cultuelle en abritant des rites de pluie comme à Niomoune, Tiobon où la pratique existe encore. Par ailleurs au cours des cérémonies d'initiation, les futurs initiés effectuent un bain purificateur dans ces sites d'eau qui sont un passage obligé avant de rejoindre le bois sacré (Thomas, 1959 ; 1965). L’initiation étant un moment de formation, le rituel autour de ces points d'eau peut apparaître comme un moyen d'imprimer dans la conscience de ces jeunes l'importance de ces sites dans la pérennisation de la tradition du village. A ce propos, Diatta et al. (2017) sont d'avis que ces sites constituent de véritables lieux de préservation des secrets et pratiques multiséculaires.

Il faut toutefois souligner que les lieux de résidence des mânes des ancêtres des ajamaat sont surtout localisés dans les mares. Les cours d'eau (bolong et fleuve) et les forêts de palétuviers sont investis par d'autres esprits des morts à savoir les koummhāl (āmmhāl au singulier) (Thomas, 1959 ; Id. 1967 ; Pognant, 1997 ; Baum, 1999 ; Diabone, 2010 ; Diédhiou, 2011). La crainte qu'ils inspirent chez l'ajamaat oblige ce dernier à ne pas se considérer " maître exclusif » des cours d'eau et de leurs ressources. Le souci de ne pas provoquer la réaction négative de ces esprits est toujours présent chez les pêcheurs et les cueilleuses d'huître. La croyance relative à cet esprit ne subsiste cependant que dans les îles du Bliss-Kassa et dans le Huluf et l'Esulalu sur la rive sud. Dans le Blouf et le Fogny, les représentations collectives ne font pas allusion à ces esprits de l'eau. Outre les mânes des ancêtres et les esprits des morts (koummhāl), on a le système totémique ajamaat qui concoure à la préservation de la biodiversité en Basse Casamance. En effet, contrairement au totem clanique connu chez la plupart des sociétés 
africaines (Butare, 2003), chez les ajamaat, la notion d'ęwūm (siwuum au pluriel) fait référence au double animal qu'est censé avoir chaque individu. Le destin de l'homme étant solidaire à celui de son ęwūm, ils sont malades ensemble, la mort de l'un entraine fatalement la mort de l'autre (Thomas, 1959 ; Journet-Diallo, 1998). Il se trouve que chez les ajamaat, l'essentiel des animaux qui incarnent cette fonction de ęwüm sont des êtres aquatiques : le crocodile (yōn), le requin (punyog), le poisson-scie (kahlum), l'hippopotame (ękāw ou ęrindaay), le lamantin (baliilaab), le varan (ebalass), etc. (JournetDiallo, 1998, Diatta et al. 2017). Or, l'habitat naturel de ces totems, alter ego des humains, reste le milieu aquatique notamment le fleuve, les bolong et les mares. C'est pourquoi les rapports entre l'ajamaat et le milieu aquatique sont empreints d'attention. Ce milieu qui apparaît comme un « deuxième monde » auquel dépend la vie des membres de la communauté est à protéger pour la sécurité et le bien-être commun. Certes, les siwuum aquatiques notamment ceux appartenant aux enfants et incapables de se protéger, résident habituellement au fond du marigot ou de la rivière (Diédhiou, 2011), mais, il arrive que certains s'éloignent de ces fonds marins et se retrouvent à la merci des filets de pêche. Ils peuvent aussi être effrayés par le bruit du moteur ou bien se retrouver dans un lieu où se récoltent des huitres. Ainsi, en pêchant dans ces endroits ou en y cueillant les huitres, les totems pourraient être capturés, blessés accidentellement (Diatta et $a l$. 2017). D’où les interdits qui frappent les activités de pêche et de cueillette d'huitres dans certains bolong de Basse Casamance (Fall et al., 2011).

Par ailleurs, dans l'imaginaire collectif ajamaat, on pense que la pérennité de l'eau dans les points d'eau abritant les totems serait liée à la présence de ces êtres mystiques. C'est pourquoi si leur quiétude est perturbée jusqu'à provoquer leur départ d'une résidence habituelle, les conséquences seront désastreuses pour la communauté. En effet, non seulement les points d'eau qui leur servaient d'habitacle s'assèchent mais leur départ de ces demeures naturelles protégées par la coutume, les expose à la menace des chasseurs et pêcheurs de même qu'à certains phénomènes destructeurs de la biodiversité comme les feux de brousses.

De nombreux sites sont également vus comme étant des demeures de génies. Dans la cosmogonie traditionnelle comme dans la pensée islamique, ces êtres surnaturels existent. En revanche, dans la pensée islamique, ces êtres surnaturels sont connus sous le nom de djinns. Le Coran en fait allusion dans l'une de ses sourates (Coran 51:56). C'est ainsi qu'avec l'islamisation de la Casamance à partir du XIXe siècle, il va apparaître des sites d'eau identifiés comme étant la demeure de ces êtres. Ces derniers sont des créatures de Dieu qui forment une sorte d'humanité parallèle et invisible et dotés de pouvoirs supérieurs (Diatta, 2018). L'on remarquera que ces sites d'eau peuplés et protégés par les djinns sont localisés dans des provinces plus islamisées 
comme le Fogny diabancouna et le Blouf. Ce qui dénote qu'en dépit de leur islamisation, les populations n'ont pas voulu rompre les rapports empreints de respect et de craintes vis-à-vis de ces sites d'eau qui représentent leur identité communautaire. Elles ont donc trouvé un moyen de conserver ces rapports en faisant cohabiter les anciens esprits et génies aux djinns ou bien en les substituant par ces derniers. Diédhiou (2001) conforte cette idée en affirmant que dans les zones islamisées du Fogny et du Blouf, les populations ont comparu les fétiches ou ukiin (singulier : boekin) aux djinns par une sorte de déplacement du sacré.

Chez d'autres sociétés négro-africaines, on relève ce même phénomène dans les rapports au milieu naturel. Ainsi chez les Lébou, Dumez et Ka (2000) expliquent qu'au cours de leurs enquêtes, les qualificatifs $r a b$ (esprits ancestraux) et djinné sont indistinctement utilisés par leurs interlocuteurs pour désigner les différents génies du panthéon lébou. Ainsi dans notre zone d'étude, l'un des exemples les plus illustratifs de ce fait est le site de la mare sacrée de Kadieel à Elana. Habité par une population à moitié islamisée, les traditions de ce village évoquent la présence d'un djinn qui a élu domicile sur un grand fromager qui surplombe le site. On rapporte que du haut de ce fromager, provient une lumière pendant certaines nuits de vendredi que les pêcheurs aperçoivent à partir du bolong d'Elana. Nous pensons par conséquent que le fait que ce site d'eau soit parmi les mieux protégés dans les parages, malgré la disparition de la mare traditionnelle, soit lié à cette sorte de syncrétisme qu'il symbolise. De nombreux autres sites d'eau de la partie nord de notre zone d'étude doivent leur protection à la présence des djinns.

Par ailleurs, outre ces paysages d'eau protégés par des djinns, il y a ceux dont la sacralité est liée à la présence de fétiche et d'autres au séjour du grand chef religieux musulman El Hadj Omar Tall. La sacralité qui entourent ces sites font que nombre d'entre eux sont sujets à des prohibitions qui contribuent à leur préservation. Le cas du site de Kegne kegne Diameng aux croyances islamiques constitue un exemple patent. Les nombreux arbres se trouvant dans l'enceinte dudit lieu sacré sont interdits à toute exploitation en dépit de sa proximité avec le quai de pêche de Kafountine, principale cause de destruction des forêts dans la zone pour le fumage du poisson (Diatta, 2018). Il semble donc que la sacralisation des paysages d'eau et leur protection est un fait caractéristique de la civilisation Ajamaat. Cette conception vis-à-vis du milieu aquatique trouve son origine dans les croyances de ce peuple. Ainsi, malgré l'évolution notée dans ces croyances avec l'adoption de nouvelles religions, les sociétés locales ont réussi à préserver les rapports privilégiés avec la nature. D’ailleurs, les croyances et mythes développés autour des paysages d'eau en font des milieux privilégiés de ponte et de refuge pour certaines espèces aquatiques de la zone (Gadou, 2003 ; Diatta et al., 2017). 


\section{Conclusion}

La religion traditionnelle ajamaat, comme toutes les croyances religieuses, place l'eau au centre de la vie sociale. Ayant peuplé un espace qualifié d' « amphibie » à cause de ses paysages d'eau, les ajamaat ont en même temps forgé une culture attachée à cet environnement aquatique. Demeure privilégiée des mânes des ancêtres, des animaux doubles totémiques et de certaines puissances invisibles de l'univers religieux ajamaat, ces paysages d'eau constituent un fondement de l'identité culturelle de cette population. Ainsi, les liens de sacralité qui unissent le ajamaat à ces paysages d'eau ont fait que leur préservation a toujours constitué une préoccupation pour la société. Milieux naturels abritant de nombreuses espèces à la fois végétales et animales, les interdits traditionnels et la crainte mystique développée autour de certains sites d'eau contribuent grandement à la protection de la biodiversité. Il faut toutefois souligner que des phénomènes liées la dégradation des conditions climatiques dont les effets sont très visibles sur certains d'entre eux, de même que la pression foncière actuelle, constituent des menaces sur la préservation de cet environnement. Il s'y ajoute les mutations socioculturelles liées à l'adoption des religions étrangères (Islam et Christianisme) qui se traduisent par une banalisation des interdits coutumiers relatifs à la protection des sites sacrés traditionnels. Ce qui peut conduire à un désintéressement progressif de la communauté exposant ainsi les ressources naturelles à une exploitation anarchique. Pourtant l'exemple des sites d'eau sacrés liés au passage d'El Hadj Omar Tall nous renseigne que les croyances traditionnelles ajamaat ne sont pas restées immuables au cours de l'histoire. L'étude a montré qu'il existe un syncrétisme entre la une religion traditionnelle ajamaat et l'islam à travers la sacralité qui leur est conférée. Ce qui a pu favoriser une certaine permanence dans la protection et la préservation de biodiversité autour de plusieurs de ces espaces d'eau.

\section{References :}

1. ANSD/SRPSZ (Service Régional des Pêches et de la Surveillance de Ziguinchor). (2015). "Situation économique et sociale régionale 2013 », 126 pages

2. Assipolo L. et Ndonko P. (2012). L'eau dans la pratique de l'ordalie : une lecture anthropologique et (socio)linguistique1, Psycause $n^{\circ} 61$, Lyon, Mario Mella Édition, pp. 37-41.

3. Bassène P.Ch. A. (2011). Histoire authentique de la Casamance. Le pays Ajamaat, influences adventives, entraves des institutions traditionnelles et manifestation de l'Etat dans la colonie française du Sénégal C.-1500 -C.1947, Toulouse, La Brochure/Injé Ajamaat.

4. Barry B. (1988). La Sénégambie du XVe au XIXe siècle. Traite négrière, Islam et conquête coloniale, Paris, L'Harmattan. 
5. Baum R.M. (1999). Shrines of the Slave Trade. Diola religion and society in precolonial Sénégambia, New York, Oxford, Oxford University Press.

6. Bertrand-Bocandé E. (1849). « Notes sur la Guinée Portugaise ou Sénégambie méridionale », in Bulletin de la Société de Géographie, tome $11, n^{\circ} 65-66$, pp. 265-350

7. Cormier-Salem M-C. (1992). Gestion et évolution des espaces aquatiques : la Casamance, ORSTOM, Collection Etudes et thèses Paris.

8. Dumez R. et Ka M. (2000). «TERRITOIRE ASSIÉGÉ YOFF. Un village lébou dans la banlieue de Dakar ». Collection Dossiers régions côtières et petites îles 7. Ed. UNESCO 2000.CNRS Programme Environnement Université Cheikh Anta Diop de Dakar - Chaire UNESCO. 81 pages. Site web: http://www.unesco.org/csi/pub/papers2/yoff.htm, téléchargé le $01 / 04 / 2020$

9. Diabone C. (2010). Les ressources foncières et forestières et le développement en Casamance : regard de l'anthropologie du développement sur l'agglomération de Houlouf, Mémoire de maîtrise en de l'Université Laval/Québec, département d'anthropologie, faculté des sciences sociales, 140 pages.

10. Diatta C.S., Diouf M., Karibuhoye C. et Sow A.A. (2017). " Sites naturels sacrés et conservation des ressources marines et côtières en milieu traditionnel diola (Sénégal) », Revue d'ethnoécologie [En ligne], 11|2017, mis en ligne le 03 juillet 2017, consulté le 03 juillet 2017. URL: http://ethnoecologie.revues.org/2900

11. Diatta C.S. (2018). «Savoirs locaux et modes traditionnels de gestion des ressources naturelles marines et côtières en BasseCasamance: perspectives de leur intégration dans le système conventionnel ». Thèse de doctorat unique. Spécialité : Géographie, option Environnement. UCAD/Dakar.

12. Diédhiou L. (2001). «Projets de développement et représentations sociales en Basse Casamance : le DERBAC et le PROGES », Thèse Ph.D en sociologie, Faculté des études supérieures, Université de Montréal, 399p.

13. Diédhiou P. (2011). L’identité jóola en question. La bataille idéologique du MFDC pour l'indépendance, Paris Karthala, 404 p.

14. Djigo A. (2001). « Patrimoine culturel et Naturel de la Réserve de Biosphère du Delta du Saloum, (RBDS) : Etude de quelques croyances, pratiques traditionnelles et stratégies de protection » Mémoire de D.E.A., UCAD. 
15. FALL R. (2013). «Le Saalum de la fin du XVe siècle au milieu du XIXe siècle. Populations, espace et histoire ", Thèse de Doctorat d'Etat es Lettres, UCAD, FLASH, Département d'Histoire, 606.pages

16. Fall S.M., Diouf M. \& Badiane S.D. (2011). Identification et Caractérisation des Sites Naturels Sacrés Côtiers et Marins en Afrique de l'Ouest. Cas du Sénégal, 39 pages

17. Gadou D.M. (2003). « Préservation de la biodiversité : les répondes des religions africaines ", in Pratiques culturelles, la sauvegarde et la conservation de la biodiversité en Afrique de l'Ouest et du Centre, CRDI/Zoom Editions, pp.47-67

18. Girard J. (1969). Genèse du pouvoir charismatique en Basse Casamance, Dakar, IFAN

19. Journet O. (1976). « Rôles et statuts des femmes dans la société diola (Basse Casamance)». Thèse de Doctorat de 3ème cycle, Université de Lyon II, 521 pages

20. Journet-Diallo O. (1998). « Un monde diffracté. Théories joola du double animal », Systèmes de pensée en Afrique noire, 15 |, 203-230, accès : Https://span.revues.org/1598. HTML visité le 15 février 2015.

21. Journet-Diallo O. (2007). Les créances de la terre. Chroniques du pays jamaat (Joola de Guinée- Bissau) : avant-propos et introduction. Bibliothèque de l'Ecole des Hautes Etudes. Les créances de la terre. Chroniques du pays jamaat (Joola de GuinéeBissau), Brepols, Turnhout, pp 9-52, collection des sciences religieuses, $\mathrm{n}^{\mathrm{o}}$ 134: https://halshs.archives-ouvertes.fr/halshs00686115

22. Linares O.F. (1992). Power, prayer and production: the Jola of Casamance, Senegal, Cambridge University Press, 258 pages

23. Oyono P.R., Fall S.M., Karibuhoye C. S., Said A.R., Sidibeh L., Sow M. (2012). Les Sites Naturels Sacrés de l'Ecorégion Côtière et Marine Ouest-Africaine. Une Exploration et des Options de Reconnaissance Institutionnelle. FIBA, Dakar, 52 pages

24. Pélissier P. (1966). Les paysans du Sénégal, Les civilisations agraires du Cayor à la Casamance, Fabrègue St Yrieux).

25. Pognant T. (1997). "Eau et êtres aquatiques sacrés en Gambie », Thèse de doctorat nouveau régime en anthropologie, Université René Descartes-Paris V, Faculté des Sciences Humaines et Sociales Sorbonne, Département de Sciences Sociales 362.

26. Pont-Humbert C. (1995). Dictionnaire des symboles, des rites et des croyances, Editions Jean-Claude Lattès, 438p. 
27. Quéchon M. (1971). «Réflexions sur certains aspects du syncrétisme dans l'islam ouest-africain ", in Cahiers d'Etudes Africaines, Vol. 11, Cahier 42, pp. 206-230.URL: http://www.jstor.com/stable/4391111

28. Tabard R. (2010). «Religions et cultures traditionnelles africaines », Revue des sciences religieuses, 84, $\mathrm{n}^{\circ}$ 2, pp. 191-205. URL : http://journals.openedition.org/rsr/346; DOI https://doi.org/10.4000/rsr.346

29. Tahirindraza H. (2015). Les tabous au service de la conservation durable de l'espace Tanalana, sud-ouest de Madagascar, Université de Toliara, Madagascar, $7 \mathrm{p}$

30. Talkeu-Tounouga C. (2000). La fonction symbolique de l'eau en Afrique noire : Une approche culturelle de l'eau, Présence Africaine Editions, Nouvelle série, No. 161/162, pp. 33-47. URL: https://www.jstor.org/stable/24352085

31. Thomas L.V. (1959). Les Diola. Analyse fonctionnelle sur une population locale de Basse Casamance, IFAN-Dakar

32. Thomas L.V. (1960). « Esquisse sur les mouvements de populations et les contacts socio-culturels en pays Diola (Basse Casamance) », in Bulletin de l'IFAN, T.XXII, sèr. $\mathrm{B}, \mathrm{n}^{0} 3-4,1960$, pp.486-508, pp.488-490; Ch. Roche, op.cit., p.31

33. Thomas L.V. (1965). "Bukut chez les Diola-Niomoun », in Notes africaines, $\mathrm{n}^{0} 108$, Octobre 1965, p.97-11

34. Thomas L.V. (1967). « La place des morts dans la société africaine traditionnelle : le culte des ancêtres ", in Notes africaines, $\mathrm{n}^{0} 113$, Janvier, pp. 1-6

35. Zahan D. (1970). Religion, spiritualité et pensées en Africaines, Paris, Payot 